\title{
Inhomogeneous Mixing in Lagrangian Cloud Models: Effects on the Production of Precipitation Embryos
}

\author{
FABIAN HOFFMANN AND TAKANOBU YAMAGUCHI \\ Cooperative Institute for Research in Environmental Sciences, University of Colorado Boulder, and \\ Chemical Sciences Division, NOAA/Earth System Research Laboratory, Boulder, Colorado \\ GRAHAM FEINGOLD \\ Chemical Sciences Division, NOAA/Earth System Research Laboratory, Boulder, Colorado
}

(Manuscript received 16 March 2018, in final form 10 October 2018)

\begin{abstract}
Although small-scale turbulent mixing at cloud edge has substantial effects on the microphysics of clouds, most models do not represent these processes explicitly, or parameterize them rather crudely. This study presents a first use of the linear eddy model (LEM) to represent unresolved turbulent mixing at the subgrid scale (SGS) of large-eddy simulations (LESs) with a coupled Lagrangian cloud model (LCM). The method utilizes Lagrangian particles to provide the trajectory of air masses within LES grid boxes, while the LEM is used to redistribute these air masses among the Lagrangian particles based on the local features of turbulence, allowing for the appropriate representation of inhomogeneous to homogeneous SGS mixing. The new approach has the salutary effect of mitigating spurious supersaturations. At low turbulence intensities, as found in the early stages of an idealized bubble cloud simulation, cloud-edge SGS mixing tends to be inhomogeneous and the new approach is shown to be essential for the production of raindrop embryos. At higher turbulence intensities, as found in a field of shallow cumulus, SGS mixing tends to be more homogeneous and the new approach does not significantly alter the results, indicating that a grid spacing of $20 \mathrm{~m}$ may be sufficient to resolve all relevant scales of inhomogeneous mixing. In both cases, droplet in-cloud residence times are important for the production of precipitation embryos in the absence of small-scale inhomogeneous mixing, either naturally due to strong turbulence or artificially as a result of coarse resolution or by not using the LEM as an SGS model.
\end{abstract}

\section{Introduction}

Clouds result from processes acting on various spatial and temporal scales, ranging from the microscopic interplay between cloud droplets and small-scale turbulence, to the global circulation, which creates the specific environments in which certain types of cloud develop (e.g., Bodenschatz et al. 2010). The tremendous range of these scales is a barrier to the scientific understanding of clouds: the available methods cannot capture the entirety of the system. This is especially evident in (but not exclusive to) numerical modeling, where limited computational resources restrict resolution, domain size, or simulated time. Based on these constraints, specific types of models have been developed, from which large-eddy simulation (LES)

Corresponding author: Fabian Hoffmann, fabian.hoffmann@ noaa.gov has emerged as the method of choice for investigating cloud processes (e.g., Cuijpers and Duynkerke 1993). These models are able to represent individual clouds, as well as entire cloud fields, and most of the relevant dynamics explicitly. However, they are restricted to resolutions of tens of meters, which do not allow the explicit representation of small-scale turbulence.

Microscale dynamics, however, are essential for the mixing between clouds and cloud-free air and their specific effects on cloud microphysics (i.e., the size and number of cloud droplets). As originally pointed out by Baker and Latham (1979), mixing can be thought of as limited by two scenarios, distinguished by their Damköhler number (Da): homogeneous and inhomogeneous mixing. The Damköhler number is defined as the ratio of a fluid time scale to a reaction time scale. In cloudphysical applications, the former is the time a turbulent eddy needs to break down to the Kolmogorov length 
scale, and the latter is either the time necessary to evaporate a droplet completely or to saturate a subsaturated volume of air by droplet evaporation. If turbulent mixing is faster than the droplet evaporation time scale, this scenario is called homogeneous mixing ( $\mathrm{Da} \ll 1)$ : all droplets experience the same subsaturation leading to the partial evaporation of all droplets while maintaining their number concentration. In the course of inhomogeneous mixing, in which turbulent mixing is slower than the evaporation of cloud droplets $(\mathrm{Da} \gg 1)$, only those droplets that experience subsaturated cloud-free air evaporate. If these droplets evaporate completely and the remainder maintain their initial size, the distribution's mean radius will be conserved while the droplet number concentration will decrease (this scenario is usually termed extreme inhomogeneous mixing). If the affected air parcel is lifted subsequently, the supersaturations it will experience are higher and the resulting diffusional growth stronger due to decreased competition for water vapor. Therefore, inhomogeneous mixing is considered as one source for the production of precipitation embryos, that is, droplets large enough to initiate collision and coalescence in warm clouds (e.g., Baker et al. 1980; Su et al. 1998; Lasher-Trapp et al. 2005). In addition to the above-introduced effects of inhomogeneous mixing, various other effects of small-scale turbulence on the initiation of rain have been investigated during the last few decades: spectral broadening by turbulent supersaturation fluctuations, enhanced collision rates due to turbulence-induced clustering of droplets, increased relative velocities, and larger collection efficiencies (e.g., Shaw 2003; Devenish et al. 2012; Grabowski and Wang 2013).

Mixing in warm clouds can be either homogeneous or inhomogeneous, but will always become homogeneous as a turbulent eddy breaks down to the Kolmogorov scale (Jensen and Baker 1989). If, however, mixing remains largely inhomogeneous during this process, cloudy and cloud-free filaments might be distinct on length scales on the order of centimeters (e.g., Beals et al. 2015), that is, substantially smaller than the typical grid spacing of current LESs. Consequently, the mixing of cloudy and cloud-free air on the LES subgrid scale (SGS) is always homogeneous since an LES cannot resolve the simultaneous presence of different air masses at the SGS; it only predicts the mean state.

The effect of inhomogeneous mixing on droplet growth can easily be parameterized by adjusting the droplet number concentration in accordance with the assumed or predicted mixing scenario (e.g., Lasher-Trapp et al. 2005; Morrison and Grabowski 2008; Jarecka et al. 2009; Hill et al. 2009; Cooper et al. 2013). But resolving inhomogeneous mixing explicitly requires reducing the grid spacing to the Kolmogorov length scale with corresponding sacrifices on the domain size and simulated time. This is actually done in computationally demanding, direct numerical simulations (DNSs), which have been used for investigating the effects of turbulent mixing processes in clouds on very limited spatial and temporal scales (e.g., Abma et al. 2013; Kumar et al. 2013, 2014; Götzfried et al. 2017).

However, it is not necessary to perform a computationally demanding DNS to reproduce the features of small-scale mixing. The so-called linear eddy model (LEM) by Kerstein (1988), a one-dimensional and hence computationally inexpensive representation of turbulence, is able to represent fundamental features of turbulence by compressing and folding the simulated domain according to the so-called triplet map, and computes the molecular diffusion of the simulated fields. The LEM is the centerpiece of the explicit mixing parcel model (EMPM) by Krueger (1993), which has been successfully used to simulate entrainment and mixing in clouds (Krueger 1993; Krueger et al. 1997; Su et al. 1998; Tölle and Krueger 2014). In fact, the EMPM (and hence the LEM) has been reported to reproduce DNS evolution of the turbulent mixing in clouds successfully (Krueger 2016).

The idea of using the LEM as a parameterization for SGS mixing in LESs for cloud-physical applications was suggested by Krueger (1993), and implementations for noncloud-physical applications have been reviewed by Menon and Kerstein (2011). In these approaches, the scalars temperature and water vapor are computed exclusively by the LEM. LES-scale resolved advection of these scalars is represented by splicing, in which certain parts of each LEM domain are exchanged between neighboring LES grid boxes. A first realization for cloudphysical applications was presented by Stechmann (2014), whose model, however, lacks the process of splicing.

Representing splicing is generally difficult. This results from the inherent unawareness of the origins of air on the LES SGS. On the edge of a cloud, for example, it is impossible to say how much air originates from the cloud or the cloud-free region, and how these fractions are arranged at the SGS. This Lagrangian information is not available in Eulerian LESs. In the following, an approach will be introduced that derives this information from a Lagrangian cloud model (LCM). LCMs are usually coupled to an LES to represent cloud microphysics by socalled superdroplets: Lagrangian particles that represent an ensemble of real droplets (Andrejczuk et al. 2008; Shima et al. 2009; Sölch and Kärcher 2010; Riechelmann et al. 2012). Here, the LCM's superdroplets will also be used to track the SGS transport of air between the LEM domains of different LES grid boxes and, therefore, resolve splicing explicitly. After a description of the 
necessary changes in the LCM, LES, and LEM, the effects of this new approach, as well as the effects of small-scale inhomogeneous mixing on the production of precipitation embryos will be discussed using an idealized two-dimensional bubble simulation, as well as a wellstudied shallow cumulus case.

\section{Model formulation}

\section{a. Lagrangian microphysics}

For this study, a simplified version of the LCM documented in Hoffmann et al. (2015), Hoffmann et al. (2017), and Hoffmann (2017) is applied, in which the processes of sedimentation and collection are neglected. The LCM is coupled to the nonhydrostatic, anelastic LES model System for Atmospheric Modeling (SAM; Khairoutdinov and Randall 2003). SAM predicts the three velocity components, liquid water static energy, water vapor mixing ratio, and the SGS turbulence kinetic energy, which is used in SAM's 1.5th-order SGS scheme (Deardorff 1980).

The microphysics in the LCM can be summarized as follows. The centerpiece of the LCM is the superdroplet, which represents an ensemble of droplets with certain properties. The most important properties are the weighting factor, which reflects the number of (identical) real droplets represented by the superdroplet; the radius of these droplets; the location of the superdroplet in the LES domain; its velocity; and a new property to parameterize SGS mixing, which will be introduced below.

The superdroplet is allowed to move anywhere in the LES model domain. Its motion is described by

$$
\frac{d X_{i, n}}{d t}=\bar{u}_{i}\left(X_{i, n}\right)+u_{i, n}^{\prime}\left[\bar{e}\left(X_{i, n}\right)\right],
$$

where $X_{i, n}$ is the location of the $n$th superdroplet in Cartesian coordinates $\left(X_{i}\right)=(X, Y, Z),\left(\bar{u}_{i}\right)=(\bar{u}, \bar{v}, \bar{w})$ is the LES-resolved velocity interpolated to the superdroplet's location [using divergence-conserving interpolation by Grabowski et al. (2018)], and $\left(u_{i, n}^{\prime}\right)=\left(u_{n}^{\prime}, v_{n}^{\prime}, w_{n}^{\prime}\right)$ is an autocorrelated stochastic velocity component calculated in accordance with the LES SGS scheme depending on the SGS turbulence-kinetic energy $\bar{e}$ to parameterize LESunresolved motions (Sölch and Kärcher 2010).

The diffusional growth of a droplet of radius $r_{n}$ is given by

$$
\left(r_{n}+r_{0}\right) \frac{d r_{n}}{d t}=G \times\left(\bar{\delta}+\delta_{n}^{\prime}\right),
$$

where $r_{0} \approx 1.86 \mu \mathrm{m}$ is a length scale associated with gaskinetic effects (Mordy 1959; Kogan 1991), $G=\left[\bar{q}_{s}\left(F_{d}+\right.\right.$ $\left.\left.F_{k}\right)\right]^{-1}$ summarizes the effects of vapor diffusion and heat conduction on condensation [for definitions of $F_{d}$ and $F_{k}$, see Rogers and Yau $\left(1989\right.$, p. 102)], and $\bar{q}_{s}$ is the temperature-dependent saturation vapor mixing ratio calculated in the LES. The absolute supersaturation of the LES grid box, in which the considered superdroplet is located at a given time, is defined as $\bar{\delta}=\bar{q}_{v}-\bar{q}_{s}$, where $\bar{q}_{v}$ is the water vapor mixing ratio predicted by the LES. Finally, $\delta_{n}^{\prime}$ is a perturbation to the absolute supersaturation determined for each superdroplet individually, and used to parameterize the effects of SGS mixing on diffusional growth as predicted by the LEM and further outlined below. The use of $\delta_{n}^{\prime}$ in Eq. (2) is similar to that in Grabowski and Abade (2017), who used it to investigate the effect of velocity-induced supersaturation fluctuations (so-called eddy hopping) on droplet growth. The initial radius of all superdroplets is set to $0.01 \mu \mathrm{m}$, and no superdroplet is allowed to evaporate any smaller.

Evaporation and condensation in the LCM feed back to the host LES by changing the water vapor mixing ratio that is calculated from the change in radius due to condensation of all superdroplets located in the same LES grid box:

$$
\begin{aligned}
\left(\frac{\partial \bar{q}_{v}}{\partial t}\right)_{\text {condensation }} & =-\frac{(4 / 3) \pi \rho_{l}}{\rho_{a} \Delta V} \sum_{n=1}^{N} A_{n} \frac{d r_{n}^{3}}{d t} \\
& =-\frac{4 \pi \rho_{l}}{\rho_{a} \Delta V} G \sum_{n=1}^{N} A_{n} \frac{r_{n}^{2}}{r_{n}+r_{0}}\left(\bar{\delta}+\delta_{n}^{\prime}\right),
\end{aligned}
$$

where $\rho_{l}$ denotes the density of liquid water, $\rho_{a}$ the density of dry air, $\Delta V$ the volume of the LES grid box, $N$ the number of superdroplets in that grid box, and $A_{n}$ the superdroplet's weighting factor.

\section{b. Using the LEM as a SGS model within an LCM-LES framework}

Before advancing to a more detailed description of the new modeling approach, the general concept of integrating the LEM into an LCM-LES framework will be discussed by comparing it to established applications of the LEM as an SGS model in standard LES (i.e., without a coupled LCM) (e.g., Menon and Kerstein 2011), which will be referred to as LES-LEM (L2) in the following. The new approach will be called LCM-LESLEM (L3). In L2, water vapor and temperature are exclusively calculated by the LEM, whose individual domains are located in each LES grid box, computing turbulent compression, folding, and molecular diffusion for the predicted fields. The LES-resolved-scale transport of water vapor and temperature is realized by splicing, in which a chunk of the LEM is removed from one LEM and added to the LEM of a neighboring LES 
grid box in accordance with the LES-resolved flow field. The new approach L3 deviates from L2 in various aspects, which will be briefly outlined here, with more details following below.

First, each superdroplet in L3 is assumed to be surrounded by a volume of air, which can be considered to be one LEM element. Accordingly, all superdroplets currently located in an LES grid box are associated with an LEM domain. Once a superdroplet moves from one LES grid box to another, this superdroplet is introduced into a new LEM domain. Thus, this approach allows one to resolve splicing explicitly; that is, it moves not only the right number of LEM elements in accordance with the LES-resolved flow field, as is done in L2, but also the correct LEM elements depending on their origin, which is inherently impossible in L2. Note that the motion of a superdroplet does not only depend on the LES-resolved flow field but also, usually to a smaller extent, on a stochastic velocity component that represents the unresolved SGS velocity fluctuations [see Eq. (1)]. These motions are unrelated to the LEM, which has no impact on the physical location of droplets. These stochastic motions are, however, essential in representing the dispersion of superdroplets in the LES domain correctly (e.g., Weil et al. 2004) and, therefore, are necessary for the correct representation of splicing between neighboring LES grid boxes.

Second, although it would be possible to use water vapor and temperature as LEM quantities in L3, they remain LES quantities. Instead, only one quantity, the perturbation of the absolute supersaturation $\delta_{n}^{\prime}$, is predicted by the LEM. This minimizes computing time and memory since only one variable instead of two are predicted by the LEM. However, this simplification potentially decreases supersaturation fluctuations resulting from the supersaturation's nonlinear dependence on temperature. Nonetheless, the LEM's general performance due to this simplification is comparable to the implementation of Su et al. (1998), which is shown in entraining parcel simulations in appendix $\mathrm{C}$.

Finally, $\delta_{n}^{\prime}$ increases or decreases the diffusional growth of droplets, but does not store water vapor and temperature in the LEM. These quantities are predicted exclusively by the LES to avoid jeopardizing their conservation by distributing them among the LES and the LEM. This is especially important since it is not easy to conserve the mass of air in each LES grid box, as in the case of L2, because of statistical fluctuations in the number of superdroplets, and therefore LEM elements, in each LES grid box (e.g., Grabowski et al. 2018). This problem becomes even more severe due to droplet sedimentation, which is why sedimentation is neglected in this study.

\section{c. Determining the supersaturation perturbation $\delta_{n}^{\prime}$}

In this study, the determination of $\delta_{n}^{\prime}$ is designed to represent SGS mixing as calculated by the LEM. We assume that each superdroplet is surrounded by its own volume of air, which can be characterized by $\delta_{n}^{\prime}$, a perturbation to $\bar{\delta}$ derived from the LES. Using three terms, $\delta_{n}^{\prime}$ is determined as follows:

$$
\frac{d \delta_{n}^{\prime}}{d t}=-\left(\frac{\partial \bar{\delta}_{n}}{\partial t}\right)_{\text {mixing,LES }}+\left(\frac{\partial \delta_{n}^{\prime}}{\partial t}\right)_{\text {mixing,LEM }}-\frac{\delta_{n}^{\prime}}{\tau_{n}} .
$$

Here, $\left(\partial \bar{\delta}_{n} / \partial t\right)_{\text {mixing,LES }}$ is the effect of the resolved-scale mixing on $\bar{\delta}$ calculated in the SGS model of the LES along the trajectory of a superdroplet (a way to determine this quantity is outlined in appendix A). Due to the negative sign, this term exactly counteracts the effect of the LES SGS model on the evolution of $\delta_{n}^{\prime}$. [Therefore, $\bar{\delta}+\delta_{n}^{\prime}$ would represent the (adiabatic) absolute supersaturation in the absence of mixing if no further terms were considered in Eq. (4).] Mixing is considered nonetheless by the second term, $\left(\partial \delta_{n}^{\prime} / \partial t\right)_{\text {mixing,LEM }}$, which depicts the SGS mixing recalculated in the LEM among all superdroplets inside an LES grid box (described in more detail in the following subsection). The last term, $-\delta_{n}^{\prime} / \tau_{n}$, represents the depletion of $\delta_{n}^{\prime}$ by condensation or evaporation. Note that this equation is calculated for all superdroplets in the entire domain. Accordingly, it is not necessary to artificially distinguish between superdroplets inside the cloud or at any other place in the LES domain.

The phase relaxation time scale $\tau_{n}$ is restricted to the volume of air surrounding each superdroplet:

$$
\tau_{n}=\left(4 \pi G^{*} \frac{r_{n}^{2}}{r_{n}+r_{0}} \frac{A_{n}}{\Delta V / N}\right)^{-1},
$$

where $\Delta V / N$ is the volume of air surrounding each superdroplet, and $G^{*}=G \Gamma \rho_{l} / \rho_{a}$, with $\Gamma=1+\left(L_{v} / c_{p}\right)\left(d q_{s} / d T\right)$ the psychrometric correction necessary to convert changes in water vapor to changes in absolute supersaturation and $d q_{s} / d T$ is the Clausius-Clapeyron relation. Due to this definition of $\tau_{n}$, the arithmetic mean of the last term in Eq. (4), calculated for all superdroplets inside a grid box and divided by $\Gamma$, corresponds exactly to the share of $\delta_{n}^{\prime}$ in Eq. (3):

$$
\frac{1}{\Gamma}\left[\frac{1}{N} \sum_{n=1}^{N}\left(-\frac{\delta_{n}^{\prime}}{\tau_{n}}\right)\right]=-\frac{4 \pi \rho_{l}}{\rho_{a} \Delta V} G \sum_{n=1}^{N} A_{n} \frac{r_{n}^{2}}{r_{n}+r_{0}} \delta_{n}^{\prime} .
$$

Accordingly, condensation and evaporation are treated consistently in the LCM and the LES.

Note that $\delta_{n}^{\prime}$ is always depleted by the third term of Eq. (4) although there might be a different stimulus 
from Eq. (2), which depends on the sum $\bar{\delta}+\delta_{n}^{\prime}$. This could be avoided by removing $\delta_{n}^{\prime}$ from Eq. (3), which would transfer water vapor and temperature to the LEM. This is not done here since it risks the conservation of water vapor and temperature by distributing them among the LES and the LEM, as explained above.

\section{d. The LEM within a Lagrangian framework}

As introduced in Eq. (4), the LEM is used to calculate the SGS turbulent rearrangement and diffusion of $\delta_{n}^{\prime}$ associated with all superdroplets inside an LES grid box. The applied LEM follows closely the implementation by Krueger (1993) and Krueger et al. (1997) in their EMPM, to which the reader is referred for more details.

Since one LEM is calculated in each LES grid box, all superdroplets currently located in that grid box are stored in the one-dimensional, cyclic domain of the LEM, in which each superdroplet represents one element of the LEM array. Accordingly, all properties of a superdroplet, and most importantly $\delta_{n}^{\prime}$, will undergo the same turbulent rearrangements described by the triplet map (described below). Moreover, the location of a superdroplet in the LEM domain is stored as a new property of each superdroplet to maintain the distribution of variables within the LEM domain over several time steps, as well as throughout the transport from the LEM of one LES grid box to another, as outlined further below.

Turbulence parameters of the LEM are set in accordance with the LES SGS model. The length of the LEM domain, denoted as $L$, which is the model integral length scale of turbulence, is set to the mixing length of the LES SGS model (which is the vertical spacing of the grid box $\Delta z$ in SAM). And the width of each LEM element, which represents one superdroplet, is

$$
\Delta z^{\prime}=\frac{L}{N}
$$

where $N$ is the total number of superdroplets in an LES grid box. Accordingly, the model Kolmogorov length scale is given by

$$
\eta=6 \Delta z^{\prime}
$$

where the factor 6 is necessary to resolve turbulent rearrangements in the LEM domain by the triplet map.

A schematic description of the LEM's workflow is presented in Fig. 1. The LEM is called after advection of the superdroplets by the LES and before the calculation of condensation and evaporation. First, a segment of the LEM domain is selected to undergo turbulent rearrangement (Fig. 1, step 1). The segment's starting
1: selection of segment for rearrangement
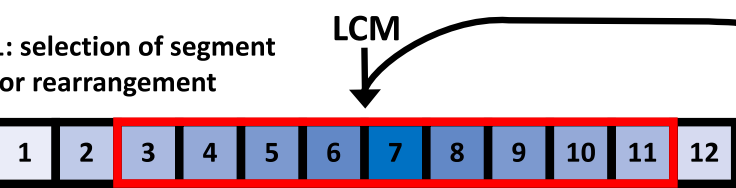

2: division in selection segments (three superdroplets each)

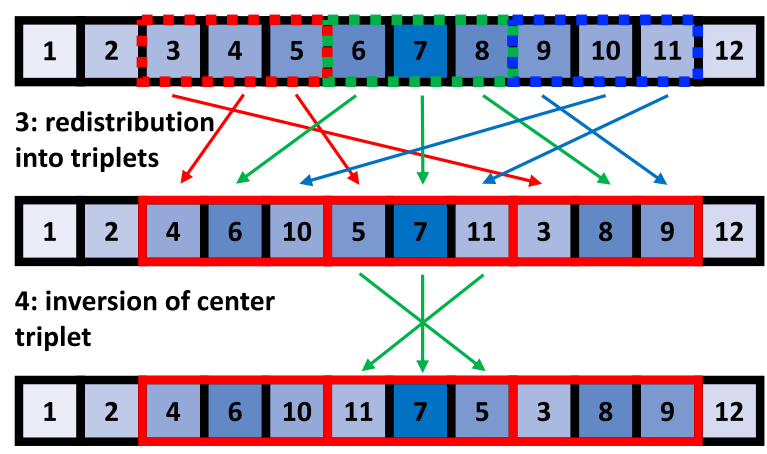

5: molecular / turbulent diffusion

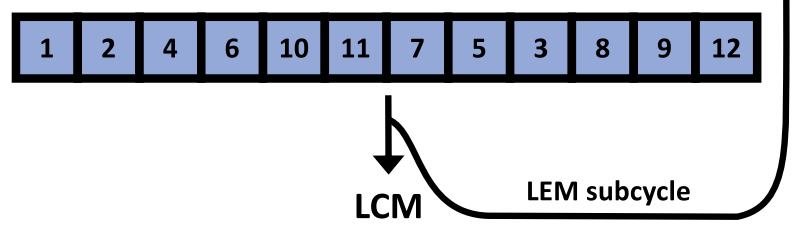

FIG. 1. Schematic representation of the LEM using superdroplets. The superdroplets are arranged in a one-dimensional array and numbered accordingly. From this array, a segment is chosen to undergo turbulent compression and folding (red line, step 1). This segment is divided into so-called selection segments of three superdroplets each (step 2), which are redistributed into thirds (triplets) of the original segment (step 3): the first entrance of each triplet comes from a red selection segment, the second from a green segment, and the third from a blue segment, which maintains the gradient of the original segment in each triplet. Then, the central triplet is inverted (step 4). Molecular (or turbulent) diffusion is applied to the entire array at the last step of the LEM (step 5).

position, as well as its length, are determined randomly, using an analytical expression for the probability of length scales in a high Reynolds number flow [see Eq. (2.3) in Krueger (1993)].

The triplet map is applied to all superdroplets in this segment [see Fig. 1 in Krueger et al. (1997)]. This is done by creating an additional array that provides space for all superdroplets in the original segment, however, divided into three partitions of (ideally) the same size (henceforth, triplet partitions). (If this is not possible without a remainder, the third triplet partition contains one superdroplet, more or less.) Then, the original segment is divided into partitions containing (ideally) three superdroplets each (henceforth selection partitions; Fig. 1, step 2). (The last segment might contain only one or two superdroplets.) Then, the superdroplets in the 
selection partitions, which are accessed successively from left to right, are randomly assigned to the triplet partitions, one superdroplet for each triplet partition, filling them successively from left to right (Fig. 1, step 3). In doing so, the general distribution of superdroplets and their properties in the original segment is maintained in each of the triplet partitions, however, compressed to one-third of the original length, which increases gradients analogous to the compressive strain in a turbulent flow. Finally, the order of the superdroplets forming the center triplet partition is reversed to represent turbulent folding (Fig. 1, step 4). Note that this variant of the spatially discrete triplet map differs from the original deterministic variant proposed by Menon and Kerstein (2011) by selecting permutations randomly, but in a systematic way, instead of a purely deterministic approach. However, both variants agree well if a sufficiently large ensemble is simulated (not shown). Note further that all properties of the superdroplets, including $\delta_{n}^{\prime}$, undergo these rearrangements prescribed by the triplet map.

Finally, molecular diffusion is applied to $\delta_{n}^{\prime}$ for all superdroplets in the same LEM domain (Fig. 1, step 5). If, however, the physical Kolmogorov scale is not resolved in the LEM, the molecular diffusion coefficient is replaced by the LEM diffusion coefficient,

$$
D_{\text {LEM }}=D_{\text {LES }}\left(\frac{\eta}{L}\right)^{4 / 3},
$$

which is scaled from the LES diffusion coefficient $D_{\text {LES }}$ calculated in the LES SGS model to match the length scales not explicitly resolved by the LEM, that is, exerting homogeneous mixing to the scales below the model Kolmogorov length scale $\eta$ (Krueger et al. 1997). Molecular diffusion constitutes the end of one LEM time step. At higher turbulence intensities, the LEM time step might be significantly smaller than the time step used by the LCM and LES. If this is the case, the LEM is subcycled. The overall change in $\delta_{n}^{\prime}$ due to processes calculated in the LEM is then considered as $\left(\partial \delta_{n} / \partial t\right)_{\text {mixing,LEM }}$ in Eq. (4).

Finally, splicing (i.e., the resolved-scale transport between the LEM domains of the different LES grid boxes) will be explained. This transport is explicitly resolved by the motion of superdroplets from one grid box to another by Eq. (1). This transport is calculated before the execution of the LEM at any time step of the LCM. Superdroplets moved from a considered grid box are simply removed from the LEM domain and the remaining elements are concatenated. Superdroplets moved to a considered grid box are treated as follows. All superdroplets originating from the same grid box are arranged in chunks. Within these chunks, the locations of superdroplets within their original grid boxes are maintained by using the aboveintroduced superdroplet property, which stores the superdroplets' LEM locations in its original domain. These chunks are then randomly concatenated, and this structure is added to a random position of the LEM domain in the LES grid box under consideration.

The computational burden of the LEM is hard to quantify a priori since it depends fundamentally on the distribution of turbulence and therefore the need for subcycling. In the cases tested here, using the LEM increased computation time by about $12 \%$ in the two-dimensional bubble case, and by $8 \%$ in the shallow cumulus intercomparison case. These cases will be presented in the following sections.

\section{Two-dimensional bubble}

\section{a. Setup}

The two-dimensional bubble test case follows Grabowski et al. (2018), to which the reader is referred for more details on the initialization. In these simulations, a bubble of saturated air is initialized in a stably stratified environment of $20 \%$ relative humidity. The bubble has a diameter of $500 \mathrm{~m}$ and relaxes to environmental values within a $100-\mathrm{m}$ radius. The domain is $3600 \mathrm{~m}$ in the horizontal direction and $2400 \mathrm{~m}$ in the vertical. An isotropic grid spacing of $20 \mathrm{~m}$ has been used throughout the domain. All simulations have been run for $1200 \mathrm{~s}$ of simulated time using a model time step of $2 \mathrm{~s}$ for the LES and the LCM; the LEM is subcycled if necessary. In all of the simulations, a cloud droplet concentration of $100 \mathrm{~cm}^{-3}$ has been prescribed, using an average number of 100,200 , or 500 superdroplets per grid box distributed randomly over the entire model domain. Simulations with and without the use of the LEM are presented, and will be referred to as LEM simulations $\left(\mathrm{LCM}_{\mathrm{LEM}}\right)$ or homogenous simulations $\left(\mathrm{LCM}_{\mathrm{hom}}\right)$, respectively. Note that the turbulence simulated in the following is twodimensional and exhibits a spurious upscale transport of energy (e.g., Boffetta and Ecke 2012), which is not present in the three-dimensional turbulence typical of clouds. However, the simulations are computationally inexpensive, which enables one to vary various parameters (especially the number of simulated superdroplets) to investigate their impact on the simulation and the mixing in the LEM.

\section{b. Results}

Figure 2 shows snapshots of the mean and standard deviation of the relative supersaturation perturbation for each grid box calculated using the LEM:

$$
\mu_{S^{\prime}}=\frac{1}{N} \sum_{n=1}^{N} \frac{\delta_{n}^{\prime}}{\bar{q}_{s}} \quad \text { and }
$$



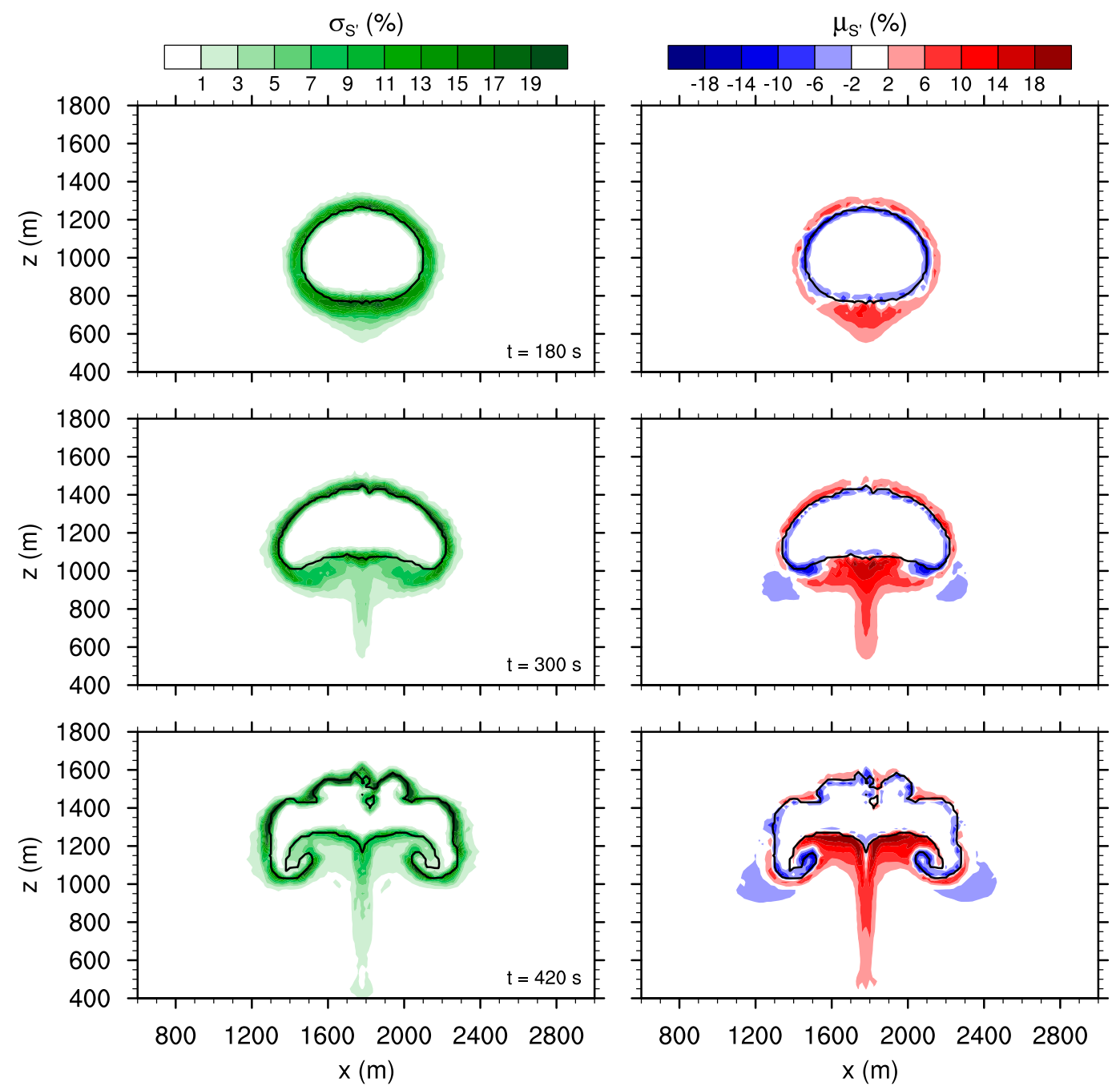

FIG. 2. (left) Variance of the supersaturation perturbation in each grid box $\sigma_{S^{\prime}}$ and (right) the corresponding mean value $\mu_{S^{\prime}}$ at simulation times of (top) 180 , (middle) 300 , and (bottom) $420 \mathrm{~s}$. Cloudy regions with a liquid water mixing ratio $q_{l}>0.01 \mathrm{~g} \mathrm{~kg}^{-1}$ are encircled by a black line. Results from the simulation using 500 superdroplets per grid box are displayed.

$$
\sigma_{S^{\prime}}=\sqrt{\frac{1}{N-1} \sum_{n=1}^{N}\left(\frac{\delta_{n}^{\prime}}{\bar{q}_{s}}-\mu_{S^{\prime}}\right)^{2}},
$$

where the saturation water vapor mixing ratio $\bar{q}_{s}$ is calculated from the LES values of temperature and pressure in the respective grid boxes.

These variables already represent the general development of the simulated cloud from a circular bubble to a turbulent cloud, as described in more detail in Grabowski and Clark (1991) and Grabowski et al. (2018). This behavior is similar in all conducted simulations. Here, only the results for the 500 superdroplets per grid-box simulation are shown.

Throughout the simulation, $\sigma_{S^{\prime}}$ exhibits a distinct maximum at the edge of the cloud (marked by a black line encircling areas with a liquid water mixing ratio $>0.01 \mathrm{~g} \mathrm{~kg}^{-1}$ ). This maximum results from cloudy filaments extending into the cloud-free regions, as well as dry environmental air that is entrained into the cloud, which are now explicitly resolved by superdroplets that either experience condensation or evaporation in an otherwise subsaturated or supersaturated grid box, respectively. This interpretation is in accord with $\mu_{S^{\prime}}$, which exhibits maxima and minima just outside and inside the cloud edge. Neither $\sigma_{S^{\prime}}$ nor $\mu_{S^{\prime}}$ is visible deeper inside the cloud due to the decreasing amount of entrained air as well as the faster depletion of sub- or supersaturations by condensation or evaporation inside the cloud. Note that $\sigma_{S^{\prime}}$ and $\mu_{S^{\prime}}$ at the top of the cloud start to fade at $420 \mathrm{~s}$ of simulated time due 

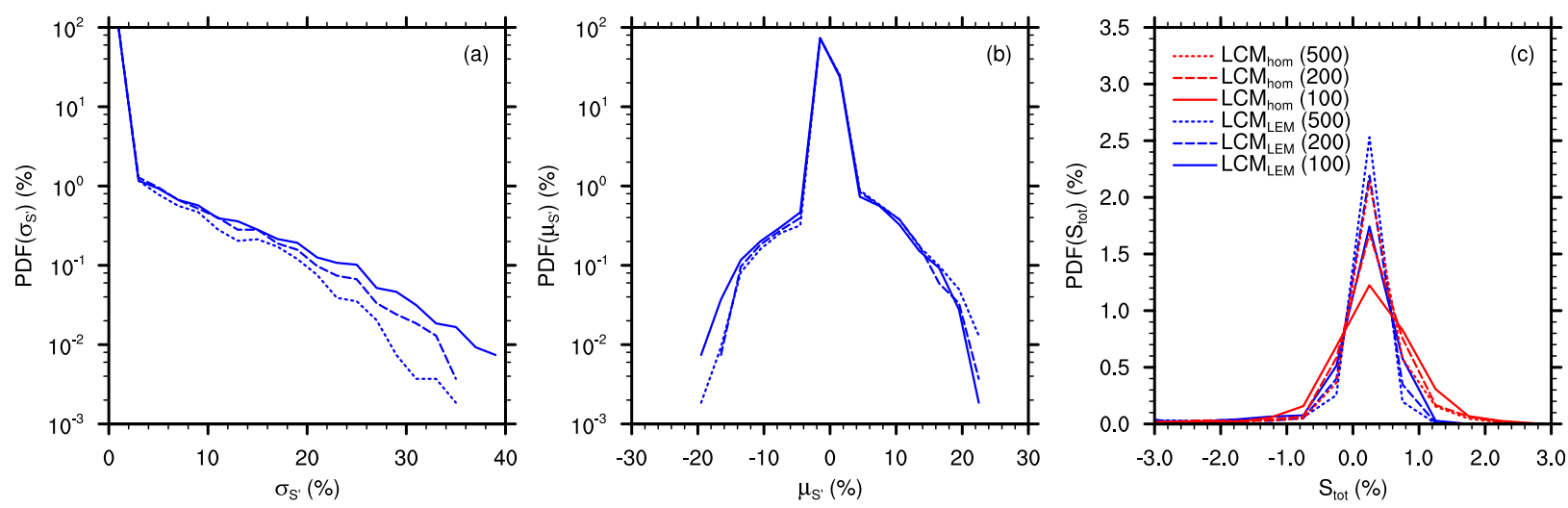

FIG. 3. PDFs of (a) the variance of the supersaturation perturbation $\sigma_{S^{\prime}}$, (b) the mean value of the supersaturation perturbation $\mu_{S^{\prime}}$, and (c) the total supersaturation $S_{\text {tot }}$ for simulations with (blue lines) and without the LEM (red lines) for superdroplet concentrations of 100, 200 , and 500 superdroplets per grid box (line patterns). The PDFs have been derived from all grid boxes at snapshots of 240-, 300-, and 360-s simulated time.

to the strong increase in turbulence with time (see also Fig. 4d), which makes the mixing more homogenous (i.e., the exchange of $\delta_{n}^{\prime}$ between the superdroplets becomes more effective). The maxima of $\sigma_{S^{\prime}}$ and $\mu_{S^{\prime}}$ below the cloud are associated with a strong downdraft that advects all scalars and accordingly superdroplets from the cloud base downward.

According to Eq. (8), the number of superdroplets per grid box is crucial in representing the Kolmogorov length scale adequately. For the simulation displayed in Fig. 2, 500 superdroplets per grid box have been used. For the applied grid spacing of $20 \mathrm{~m}$, this superdroplet concentration corresponds to a model Kolmogorov length scale of $0.24 \mathrm{~m}$, but even larger model Kolmogorov length scales might be used in typical applications, which apply about 100 superdroplets per grid box. The general dependence of $\sigma_{S^{\prime}}$ and $\mu_{S^{\prime}}$ on the number of superdroplet per grid box is displayed in the probability density functions (PDFs) in Figs. 3a and 3b, respectively. For the current model, using Kolmogorov length scales from 0.24 to 0.6 and to $1.2 \mathrm{~m}$ for 500,200 , and $100 \mathrm{su}-$ perdroplets per grid box, respectively, there are no significant deviations in the PDFs. Indeed, the shapes of the PDFs are for the most part identical, only the PDF of $\sigma_{S^{\prime}}$ exhibits slightly smaller extreme values, which are attributed to the better statistics using a higher number of superdroplets per grid box. This general agreement of the bulk properties $\sigma_{S^{\prime}}$ and $\mu_{S^{\prime}}$ for the analyzed superdroplet concentrations is in consensus with Krueger et al. (1997), who reported that results become statistically independent of the model Kolmogorov scale if it is smaller than $3 \%$ of the model integral length scale of turbulence. This criterion is fulfilled for 500 and 200 superdroplets per grid box and only mildly violated for 100 superdroplets.
Figure $3 \mathrm{c}$ displays PDFs of the total supersaturation $S_{\text {tot }}$. In simulations without the LEM (red lines), $S_{\text {tot }}$ equals the relative supersaturation calculated in the LES. In simulations with the LEM (blue lines), $S_{\text {tot }}$ is the sum of LES relative supersaturation and $\mu_{S^{\prime}}$. In all cases, the PDFs peak at a supersaturation of about $0.2 \%$, and fall off quickly toward higher and smaller values. Generally, the width of the PDF becomes narrower if the number of superdroplets per grid box is increased. These fluctuations in $S_{\text {tot }}$ are related to statistical fluctuations of the cloud droplet number concentration caused by varying the numbers of superdroplets inside a grid box. Since the statistical fluctuation of the superdroplet concentrations decreases for higher superdroplet concentrations (Grabowski et al. 2018), fluctuations of $S_{\text {tot }}$ decrease in a similar fashion. Moreover, the PDFs of $S_{\text {tot }}$ calculated from the LEM simulations are narrower than those of the corresponding homogeneous simulations. This is related to reduced spurious supersaturations, which are caused by the artificial evaporation followed by artificial condensation when a cloud moves across the numerical grid (Stevens et al. 1996; Hoffmann 2016). By using the expression for $\left(\partial \delta_{n} / \partial t\right)_{\text {mixing,LES }}$ presented in appendix A, spurious supersaturation can be significantly reduced, as outlined in more detail in appendix B.

We display the time series of the liquid water path (LWP; Fig. 4a), mean effective radius $r_{\text {eff }}$ (Fig. 4b), mean number concentration of droplets with a radius larger than $1 \mu \mathrm{m} N_{c}$ (Fig. 4c), and in-cloud integrated dissipation rate $\varepsilon$ ( Fig. $4 \mathrm{~d}$ ). At the beginning of the simulation $(<300 \mathrm{~s}$ of simulated time), LWP and $\varepsilon$ are similar for all simulations, while $r_{\text {eff }}$ is slightly larger and $N_{c}$ is smaller in the LEM simulation compared to the homogeneous simulations. This is a typical feature of inhomogeneous mixing, which is favored due to the comparably weak 

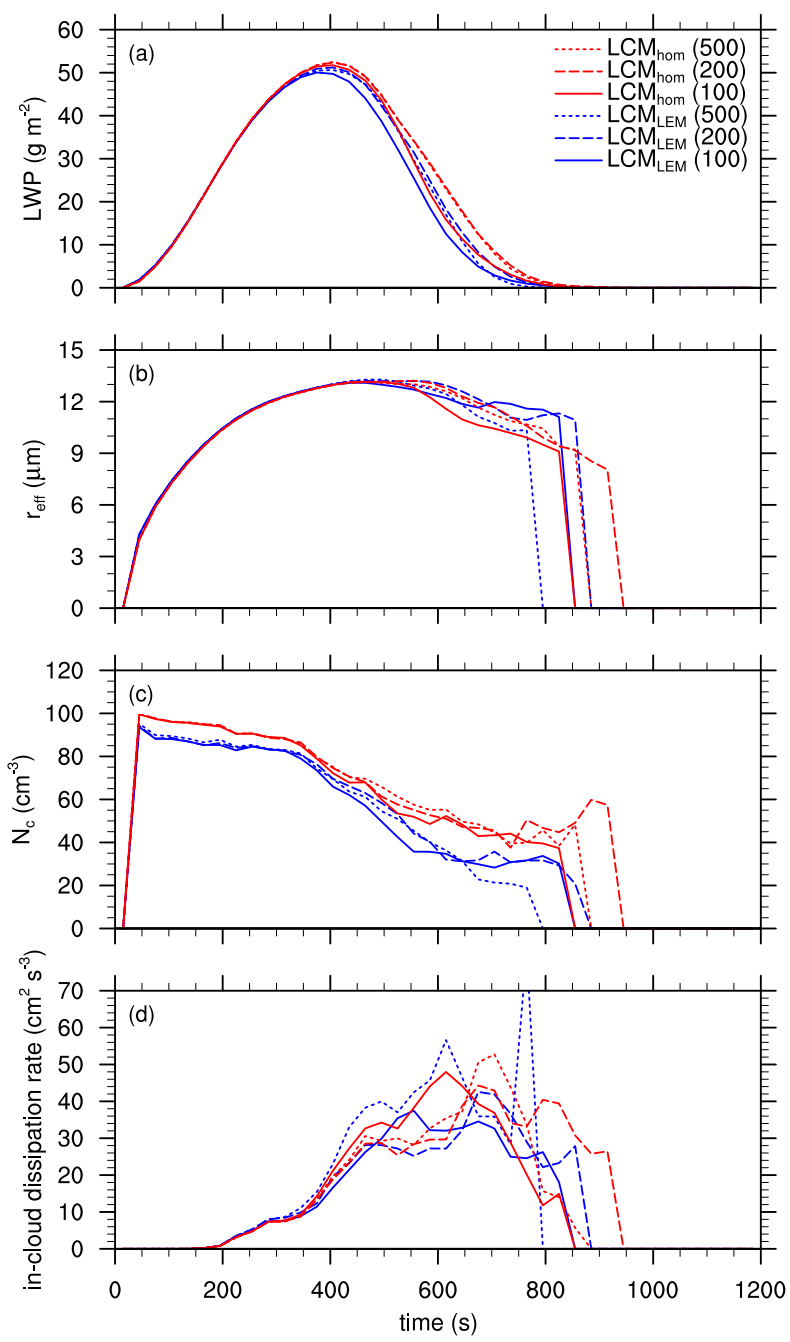

FIG. 4. Time series of (a) LWP, (b) mean effective radius, (c) droplet concentration, and (d) in-cloud dissipation rate for simulations with (blue lines) and without (red lines) the LEM for superdroplet concentrations of 100,200, and 500 superdroplets per grid box (line patterns). The quantities in (b)-(d) have been derived from columns with visible optical thicknesses exceeding 1 .

turbulence at this early stage. All in all, the changes in $r_{\text {eff }}$ and $N_{c}$ result in a longer phase relaxation time scale for the LEM simulations, which decelerates the depletion of supersaturations, causing a lower LWP, as becomes apparent after $300 \mathrm{~s}$ of simulated time (see also Hill et al. 2009). Later, $\varepsilon$ increases significantly in all simulations, resulting in a more homogeneous mixing scenario. However, due to the nonlinearity of the flow, the individual simulations start to diverge, and a direct comparison of the later stages in the cloud's development is difficult and is omitted.

In Fig. 5, PDFs of droplet radii at 300-s simulated time are displayed. The mode at $12 \mu \mathrm{m}$ can be related to the majority of the droplets inside the cloud experiencing

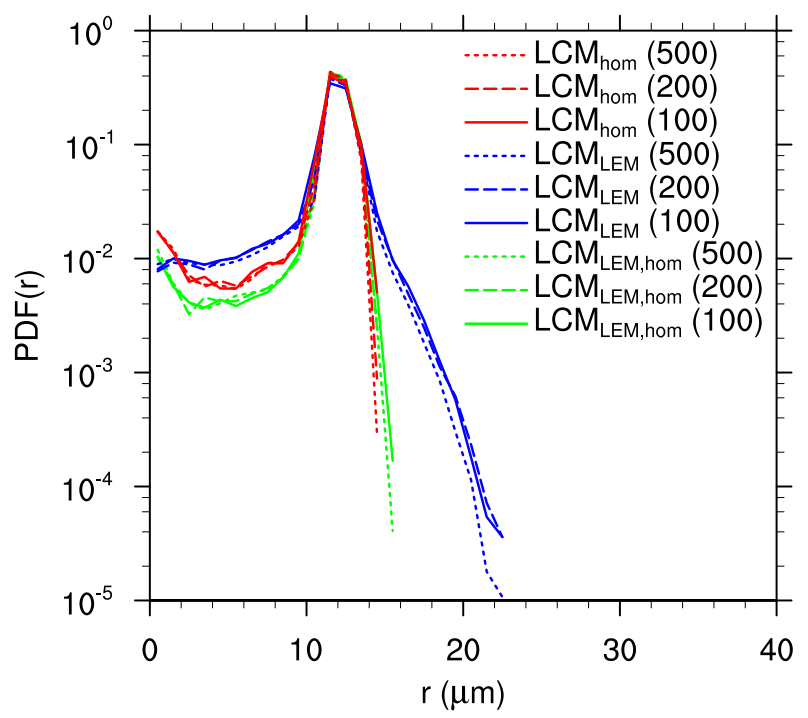

FIG. 5. PDF of the droplet radius for simulations without the LEM (red lines), with the LEM (blue lines), and with the LEM using a high turbulent diffusion coefficient mimicking homogeneous mixing (green lines) for superdroplet concentrations of 100, 200, and 500 superdroplets per grid box (line patterns) at 300-s simulated time.

near-adiabatic conditions. A second mode is visible for the homogeneous simulations (red lines) at the left edge of the spectrum $(\sim 1 \mu \mathrm{m})$ representing the droplets that have experienced strong evaporation. The LEM simulations (blue lines) deviate in two respects. First, they exhibit significantly larger droplets, and second, the distribution on the left-hand side of the adiabatic mode declines more slowly than in the homogeneous simulations. This can be attributed to the explicitly resolved SGS mixing using the LEM, which is, on the one hand, able to produce precipitation embryos due to inhomogeneous mixing as well as a smooth spectrum down to the smallest droplets. These findings resemble the results of Su et al. (1998), who used the LEM in the EMPM with an explicit representation of cloud droplets. Contrary to Su et al. (1998), who reported the necessity of resolving the physical Kolmogorov length scale, the results presented here seem to be hardly affected by the degree to which the physical Kolmogorov length scale is resolved, which is about $1.6 \mathrm{~mm}$ at $300 \mathrm{~s}$ and even smaller afterward. However, the corresponding transition length scale from inhomogeneous to homogeneous mixing introduced by Baker et al. (1980) and further investigated by Lehmann et al. (2009) is

$$
l_{*}=\varepsilon^{1 / 2} \tau_{\text {react }}^{3 / 2},
$$

which is about $0.21 \mathrm{~m}$ at $300 \mathrm{~s}$ (using a kinetic energy dissipation rate of $\varepsilon=10 \mathrm{~cm}^{2} \mathrm{~s}^{-3}$ and a phase relaxation time scale of $\tau_{\text {react }} \approx 3.5 \mathrm{~s}$ ). This length scale is of the 

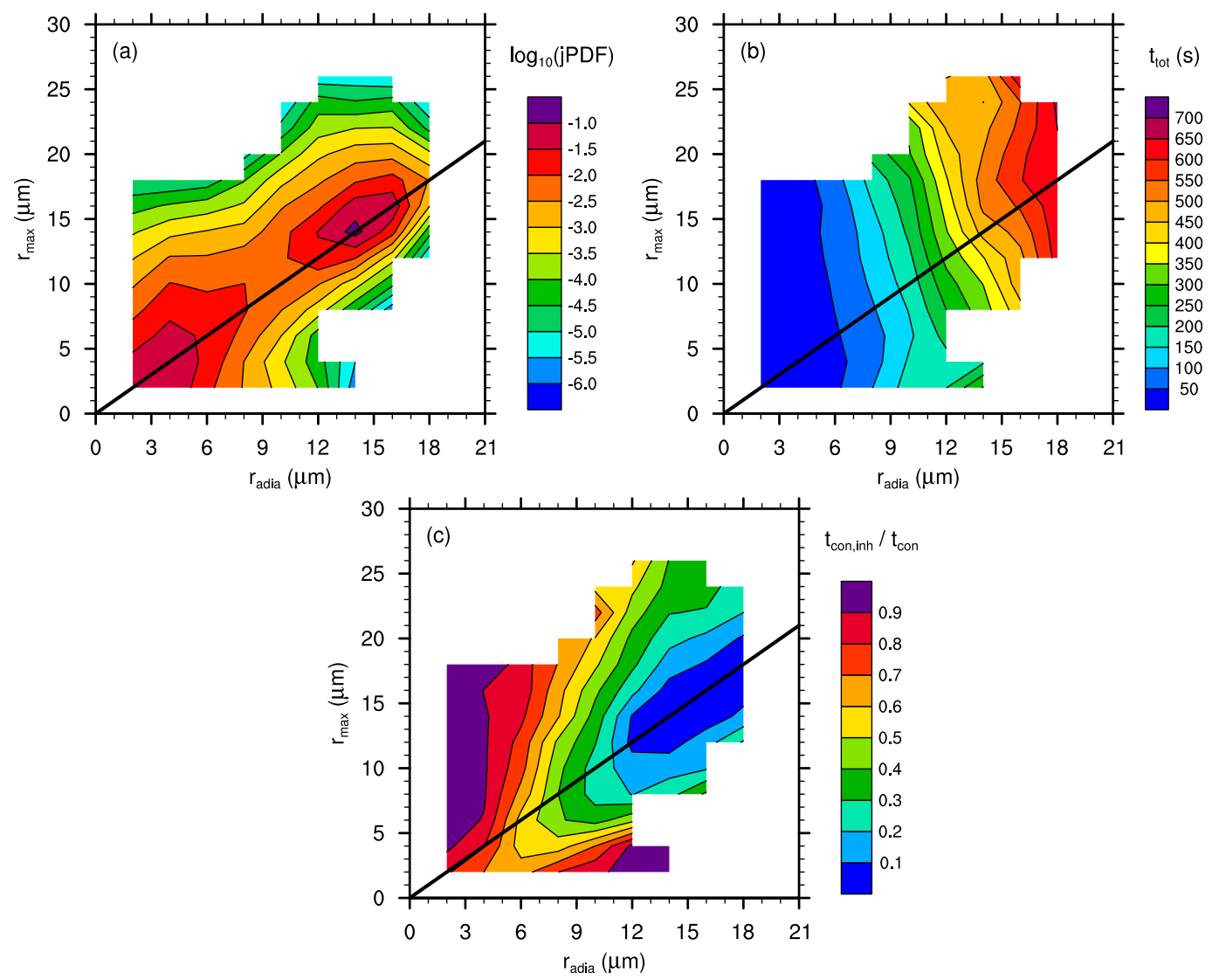

FIG. 6. (a) The joint PDF (jPDF) for the maximum radius $r_{\max }$ and (b) the corresponding adiabatic radius $r_{\text {adia }}$, as well as (c) the time spent between entrainment and reaching the maximum radius $t_{\text {tot }}$ and the time fraction of condensational growth in inhomogeneous conditions $\left(t_{\text {con,inh }} / t_{\text {con }}\right)$ as a function of $r_{\max }$ and $r_{\text {adia }}$ for all superdroplets within the LEM simulation (500 superdroplets per grid box). The thick black line marks adiabatic conditions $\left(r_{\text {max }}=r_{\text {adia }}\right)$.

same order of magnitude as the applied model Kolmogorov length scales and, therefore, justifies the homogeneous mixing happening in the LEM at scales below the model Kolmogorov length scale.

Additionally, Fig. 5 shows PDFs of the simulations using the LEM with an artificially increased turbulent diffusion coefficient of $K_{h} \approx 1800 \mathrm{~m}^{2} \mathrm{~s}^{-1}$ (corresponding to a dissipation rate of $1000 \mathrm{~m}^{2} \mathrm{~s}^{-3}$ ), which forces the LEM to represent extreme homogeneous mixing. Indeed, the general shape of these PDFs (green lines) is the same as those of the homogeneous simulations (red lines), which shows that the LEM is able to reproduce SGS homogeneous mixing at a sufficiently high turbulence intensity.

Besides small-scale inhomogeneous mixing, the incloud residence time of a droplet is assumed to be an important factor for the production of precipitation embryos.

We show the joint PDF (jPDF) for the maximum radius $r_{\max }$ and the corresponding adiabatic radius $r_{\text {adia }}$
(Fig. 6a), as well as the residence time measured as time spent between entrainment and the attainment of the maximum radius $t_{\text {tot }}$ (Fig. 6b), and the time fraction of condensational growth in inhomogeneous conditions $\left(t_{\text {con,inh }} / t_{\text {con }}\right)$ as a function of $r_{\max }$ and $r_{\text {adia }}$ (Fig. 6c) determined for each superdroplet in the LEM simulation using 500 superdroplets per grid box. The adiabatic radius $r_{\text {adia }}$ is derived from the adiabatic liquid water content produced from the superdroplet's vertical displacement between the height of entrainment into the cloud and the height of an observation, the pressure and temperature at entrainment height, and the prescribed droplet concentration of $100 \mathrm{~cm}^{-3}$. Figure 7 , derived from the corresponding homogeneous simulation, displays the first two quantities.

In general, the jPDFs (Figs. 6a and 7a) consist of two peaks: one centered at $r_{\text {max }}=r_{\text {adia }} \approx 14 \mu \mathrm{m}$ and one at the lowest displayed values of $r_{\text {max }}=r_{\text {adia }}=2 \mu \mathrm{m}$. These peaks represent droplets in the adiabatic core of the cloud as well as droplets at the edge of the cloud before 

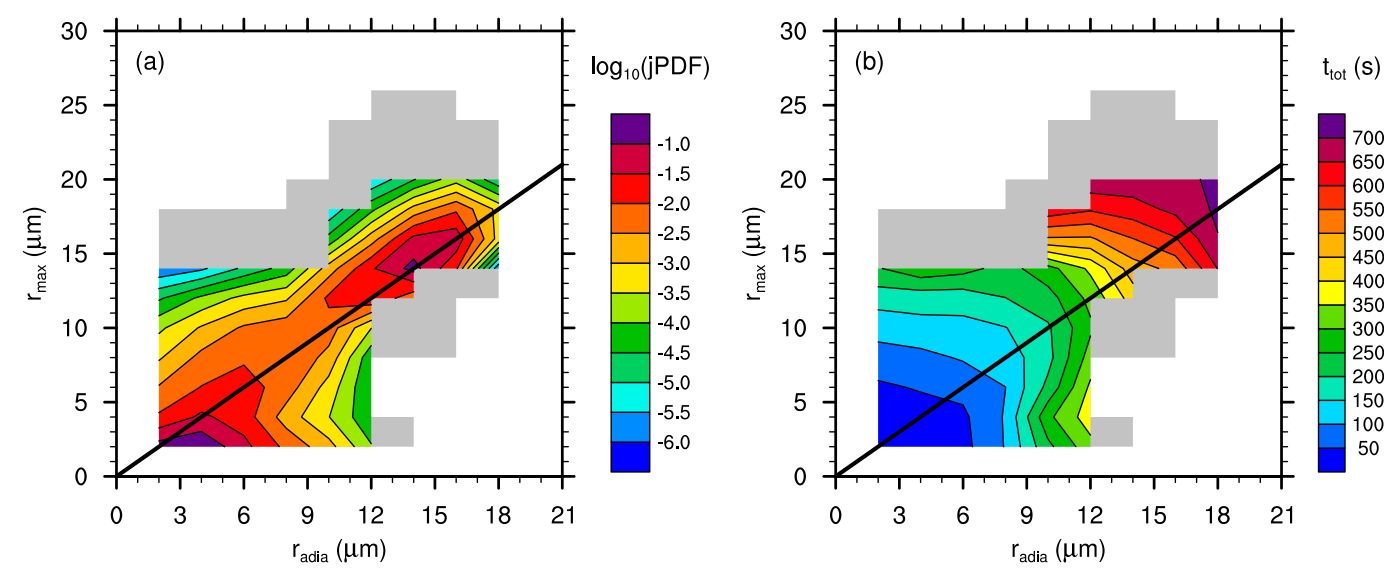

FIG. 7. (a) The joint PDF (jPDF) for the maximum radius $r_{\max }$ and the corresponding adiabatic radius $r_{\text {adia }}$, as well as (b) the time spent between entrainment and reaching the maximum radius $t_{\text {tot }}$ as a function of $r_{\max }$ and $r_{\text {adia }}$ for all superdroplets within the homogeneous simulation (500 superdroplets per grid box). The shaded area displays the range covered in the LEM simulation (see Fig. 6). The thick black line marks adiabatic conditions $\left(r_{\text {max }}=r_{\text {adia }}\right)$.

complete evaporation, respectively. Most other droplets are located in adiabatic conditions $\left(r_{\max }=r_{\text {adia }}\right)$, while the number of nonadiabatic droplets decreases rapidly. However, the jPDF is significantly broader to both super- and subadiabatic radii in the LEM simulation with a maximum droplet radius of $26 \mu \mathrm{m}$, while the homogeneous simulation exhibits a maximum radius of $20 \mu \mathrm{m}$.

The distribution of $t_{\mathrm{tot}}$ (Figs. 6b and 7b) shows distinct differences between the LEM and the homogeneous simulation. In the LEM simulation, $t_{\text {tot }}$ is almost entirely determined by $r_{\text {adia }}$ and therefore the time necessary to reach a certain height inside the cloud. Accordingly, inhomogeneous mixing must be responsible for the accelerated growth of these droplets. In the homogeneous simulation, however, the development of superadiabatic droplets depends heavily on the time spent inside the cloud. In fact, for sufficiently large superadiabatic droplets, $r_{\max }$ is only a function of $t_{\mathrm{tot}}$, which indicates that the residence time of a cloud droplet becomes essential for the production of precipitation embryos in the absence of small-scale inhomogeneous mixing. Note, however, that conclusions regarding droplet residence times need to consider the fact that the applied model neglects sedimentation, which might underestimate mixing ( $\mathrm{Su}$ et al. 1998; Tölle and Krueger 2014) and therefore potentially overestimates the time spent in environments conducive to drizzle formation.

The time spent in inhomogeneous condensational growth is determined as the time in which a superdroplet experiences condensational growth while other superdroplets in the same grid box experience evaporation, shown as $t_{\text {con,inh }} / t_{\text {con }}$ in Fig. $6 c$. This quantity is used as a proxy for the positive effect of inhomogeneous mixing on droplet growth, in which evaporation reduces the number of cloud droplets and therefore accelerates the condensational growth of the remaining droplets. However, it misses environments in which inhomogeneous mixing might not result in simultaneous condensation and evaporation. The distribution of $t_{\text {con,inh }} / t_{\text {con }}$ shows that almost all droplets outside the adiabatic core experience inhomogeneous mixing, and the largest contribution of inhomogeneous condensational growth is observed for (i) the smallest adiabatic radii, which are potentially close to the edge of the cloud where mixing takes place, and (ii) the largest superadiabatic droplets, which need these conditions to grow to such sizes. Interestingly, this applies also to subadiabatic droplets, for which total evaporation might be prevented by exposure to cloudy, supersaturated filaments for a longer time due to the resolved SGS inhomogeneous mixing.

The overall decreasing impact of inhomogeneous mixing on condensation growth at larger $r_{\text {adia }}$ can be related to the longer time these droplets have spent inside the cloud and accordingly the higher degree of turbulence that develops (Fig. 4d), resulting in a faster homogenization of air masses.

\section{Shallow cumulus test case}

\section{a. Setup}

The shallow cumulus test case follows the LES intercomparison by Siebesma et al. (2003), who developed this case based on the Barbados Oceanographic and Meteorological Experiment (BOMEX) measurement campaign (Holland and Rasmusson 1973). Similar to 

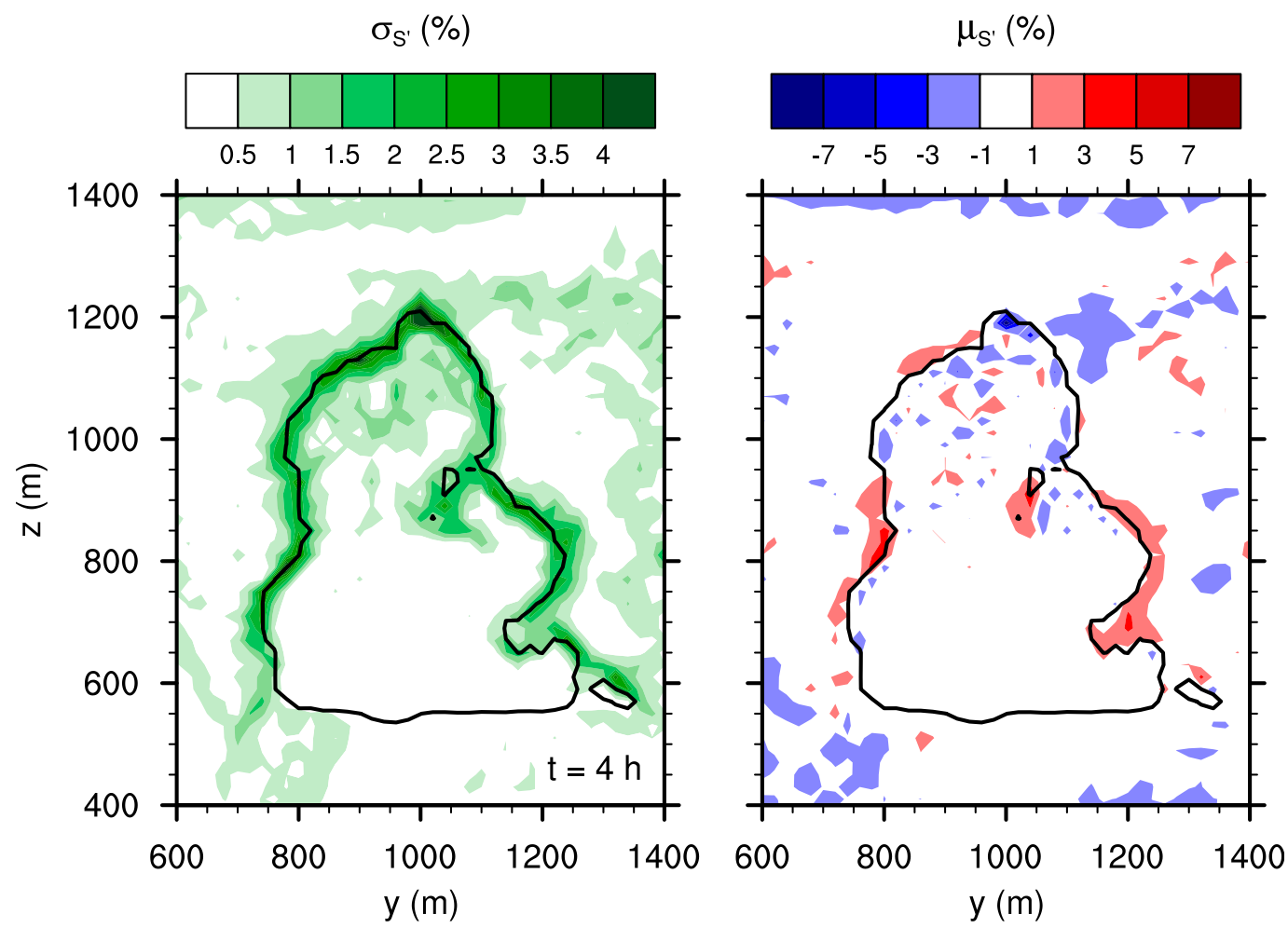

FIG. 8. (left) Variance of the supersaturation perturbation $\sigma_{S^{\prime}}$ and (right) the corresponding mean value $\mu_{S^{\prime}}$ for a randomly selected cloud at 4-h simulation time. Cloudy regions with a liquid water mixing ratio $q_{l}>0.01 \mathrm{~g} \mathrm{~kg}^{-1} \mathrm{are}$ encircled by a black line.

Siebesma et al. (2003), a $6.4 \times 6.4 \times 3.5 \mathrm{~km}^{3}$ model domain is simulated, using the same initial profiles, surface fluxes, and large-scale forcings. The only deviation is the grid spacing, which has been reduced to $20 \mathrm{~m}$ isotropically. A model time step of $2 \mathrm{~s}$ is used for the LES and the LCM; the LEM is subcycled as necessary. The simulated time is $6 \mathrm{~h}$, with analysis focused on the last $4 \mathrm{~h}$. The LCM is initialized with 100 superdroplets per grid box, representing a cloud droplet concentration of $100 \mathrm{~cm}^{-3}$. The resulting model Kolmogorov length scale of $1.2 \mathrm{~m}$ is significantly larger than the physical Kolmogorov length scale of about $1 \mathrm{~mm}$ in the highly turbulent environment of cumulus clouds. A smaller model Kolmogorov length scale is hence desirable but the necessary concentration of superdroplets is computationally unfeasible. However, the transition length scale from inhomogeneous to homogeneous mixing is about $0.83 \mathrm{~m}$ for this case [Eq. (12) using a kinetic energy dissipation rate of $\varepsilon=25 \mathrm{~cm}^{2} \mathrm{~s}^{-3}$ and a phase relaxation time scale of $\left.r_{\text {react }}=6.5 \mathrm{~s}\right]$. Accordingly, the applied model Kolmogorov length scale might be sufficient for representing the essential dynamics of inhomogeneous mixing successfully, while mixing on smaller length scales is homogeneous anyhow. Again, simulations with and without the LEM are presented.
They will be referred to as the LEM ( $\left.\mathrm{LCM}_{\mathrm{LEM}}\right)$ or homogeneous simulation $\left(\mathrm{LCM}_{\mathrm{hom}}\right)$, respectively.

\section{b. Results}

The general distribution of $\sigma_{S^{\prime}}$ and $\mu_{S^{\prime}}$ in the shallow cumulus test case is exemplified by a randomly selected cloud in Fig. 8. As in Fig. 2, maxima of $\sigma_{S^{\prime}}$ are located on the cloud edge where cloudy and cloud-free air are mixed. Equally, $\mu_{S^{\prime}}$ exhibits a similar distribution as depicted above (i.e., maxima just outside the cloud and minima inside). However, the values of $\sigma_{S^{\prime}}$ and absolute values of $\mu_{S^{\prime}}$ are much smaller compared to the bubble test case resulting from the smaller supersaturation difference between the cloud and its environment (about $10 \%$ in contrast to $80 \%$ in the bubble test case) but they also indicate a faster homogenization of $\delta_{n}^{\prime}$ within each grid box due to the generally stronger turbulence. The weakened patterns of $\sigma_{S^{\prime}}$ and $\mu_{S^{\prime}}$ outside the cloud are signatures of former clouds that diffuse and vanish with time or result from the mixing between different parts of the boundary layer.

We show time series of LWP (Fig. 9a), cloud cover, determined as the fraction of columns with visible optical thickness exceeding 1 (Fig. 9b), $r_{\text {eff }}$ (Fig. 9c), $N_{c}$ (Fig. 9d), and in-cloud integrated $\varepsilon$ (Fig. 9e). For both 

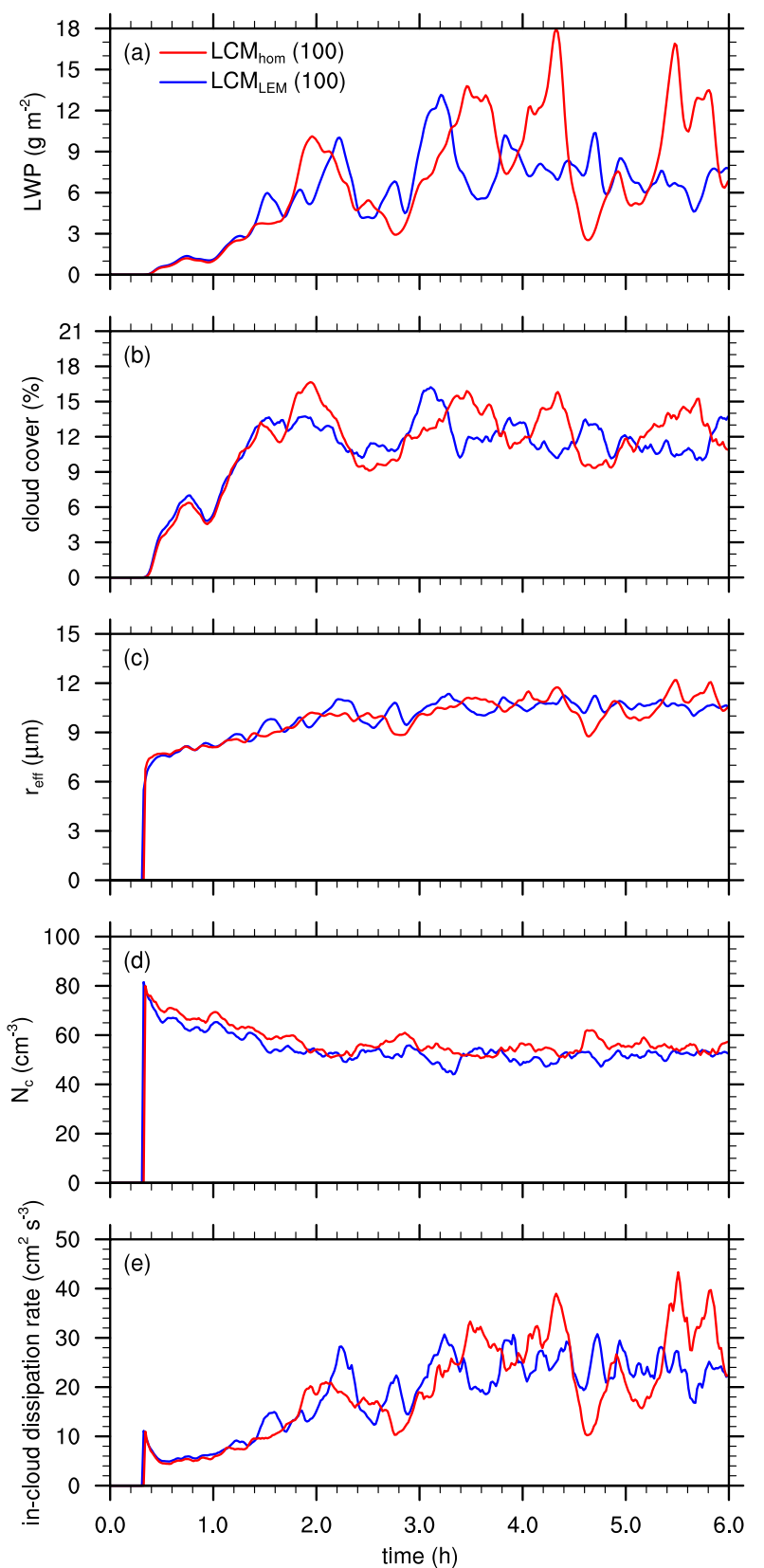

FIG. 9. Time series of (a) LWP, (b) cloud cover, (c) mean effective radius, (d) mean droplet number concentration, and (e) incloud dissipation rate for simulations without the LEM $\left(\mathrm{LCM}_{\mathrm{hom}}\right.$; red lines) and with the LEM (LCM $\mathrm{LEM}_{\mathrm{LE}}$; blue lines). The quantities in (b)-(e) have been derived from columns with visible optical thicknesses exceeding 1 .

simulations, LWP, cloud cover, $r_{\text {eff }}$, and $\varepsilon$ agree in a statistical sense. Only $N_{c}$ is about $6 \%$ smaller in the LEM simulation, which indicates a smaller impact of inhomogeneous mixing than in the bubble cloud. In fact, the general agreement, besides $N_{c}$, suggests that the additionally resolved SGS inhomogeneous mixing has a negligible impact on the general parameters of the cumulus-topped boundary layer. This expression is supported by the domain-averaged profiles of the liquid water potential temperature, the total water mixing ratio, and the conditionally averaged profiles of the cloud and cloud core liquid water mixing ratio in Figs. 10a-c, which do not exhibit significant differences. (Note that the profiles of the liquid water mixing ratio are affected by statistics due to the limited number of clouds overshooting the inversion at $1500 \mathrm{~m}$.)

Figures 11 and 12 show an analysis of superdroplet trajectories to analyze jPDFs for $r_{\max }$ and $r_{\text {adia }}$ (Figs. 11a and 12a), residence time (Figs. $11 \mathrm{~b}$ and $12 \mathrm{~b}$ ), and inhomogeneous condensational growth (Fig. 12c) similar to the analysis carried out for the bubble cloud in Figs. 6 and 7. Here, the analysis has been restricted to superdroplets that entered the cloud below $900 \mathrm{~m}$ (i.e., entrainment at cloud base). Accordingly, laterally entrained particles have been excluded from the following analysis since their adiabatic radii cannot be aligned with the vertical structure of the cloud.

The jPDF is peaked for adiabatic droplets $\left(r_{\text {max }}=r_{\text {adia }}\right)$ and falls off quickly to super- and subadiabatic droplets (Figs. 11a and 12a). Overall, the maximum radii of superadiabatic droplets are about $2 \mu \mathrm{m}$ larger in the LEM simulation compared to the homogeneous simulation. However, this difference is significantly smaller than in the bubble cloud simulations.

Inhomogeneous condensational growth (Fig. 11c) only contributes significantly to two groups of droplets. First, droplets with a small adiabatic radius $\left(r_{\text {adia }}<5 \mu \mathrm{m}\right)$ or, second, droplets with a small maximum radius $\left(r_{\max }\right.$ $<5 \mu \mathrm{m})$. Although droplets of the second group have been lifted inside the cloud, their overall growth is subadiabatic. This indicates that these droplets must have, at some point, been located at the cloud edge where mixing takes place and dilution diminishes the overall droplet growth, although it is inhomogeneous for a certain period of time. Droplets in the first group did not experience significant lifting. Accordingly, they must have been located at the cloud base for a longer time, where condensational growth can be inhomogeneous for a longer time. This pattern can be related to the general increase in turbulence in cumulus clouds, where the dissipation rate increases from negligible values at cloud base to values of $300 \mathrm{~cm}^{2} \mathrm{~s}^{-3}$ at cloud top [e.g., Fig. 14 in Hoffmann et al. (2014)]. Thus, intense turbulence effectively homogenizes differential $\delta_{n}^{\prime}$ at greater heights and therefore inhibits any inhomogeneous condensational growth aloft. At cloud base, where turbulence is weak, differences in $\delta_{n}^{\prime}$ might persist longer and enable inhomogeneous growth.

In general, the distribution of the residence time $t_{\text {tot }}$ is similar for the LEM and the homogeneous simulation 

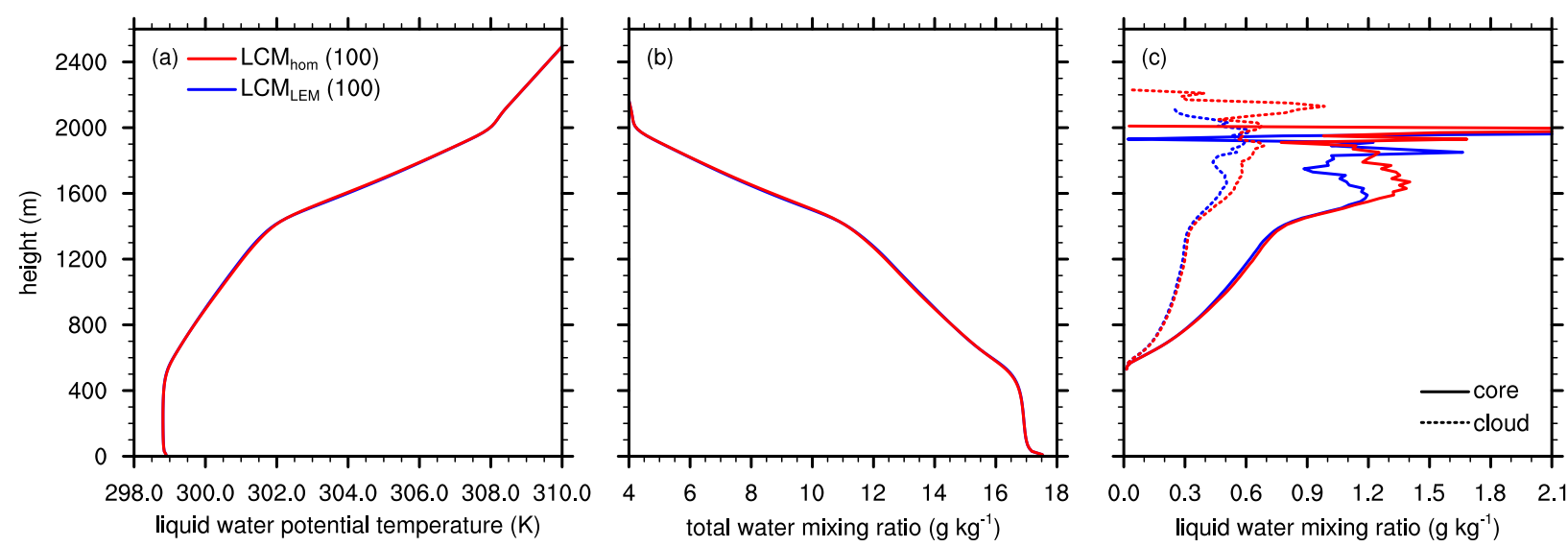

FIG. 10. Domain-averaged profiles of (a) liquid water potential temperature, (b) total water mixing ratio, and (c) conditionally averaged

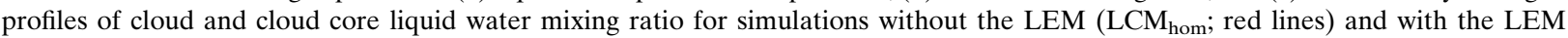
( $\mathrm{LCM}_{\mathrm{LEM}}$; blue lines). The profiles have been averaged over the last $4 \mathrm{~h}$ of the simulation.

(Figs. 11b and 12b). Only for droplets that experienced significant inhomogeneous condensational growth is $t_{\text {tot }}$ decreased, which indicates that inhomogeneous condensational growth accelerates droplet growth in the LEM simulations. But this is restricted to smaller adiabatic radii (i.e., at lower levels of the cloud). At higher levels, $t_{\text {tot }}$ does not show significant differences between the simulations. Accordingly, the residence time is more important for the production of precipitation embryos in these highly turbulent clouds, which homogenizes inhomogeneous structures quickly. Furthermore, inhomogeneous mixing is restricted to the cloud base where the lowest turbulence is located. However, this slight acceleration in droplet growth might be decisive in the initiation of the precipitation process for sufficiently long residence times.

An additional interesting finding in Fig. 12b is the existence of droplets with a lifetime of more than $60 \mathrm{~min}$ although the typical lifetime of the simulated clouds is reported to range between 10 and $40 \mathrm{~min}$ (e.g., Jiang et al. 2006). Whether these droplets where just lucky enough to be located in an extraordinarily long-living cloud or if there is another mechanism responsible, will be investigated in a future study.

\section{Conclusions}

This study presents an implementation of the linear eddy model (LEM) by Kerstein (1988) as a subgrid-scale (SGS) model to parameterize the mixing of air masses and its effects of the diffusional growth on cloud droplets in large-eddy simulations (LESs) with a coupled Lagrangian cloud model (LCM). So-called superdroplets, for which cloud microphysics are calculated in the LCM, are used to provide the history of air masses in each LES grid box. Based upon this information, a supersaturation perturbation is derived for each superdroplet, and redistributed among all superdroplets in a grid box using the LEM in accordance with the LES SGS model. This enables the appropriate representation of SGS mixing scenarios, potentially ranging from inhomogeneous to homogeneous, but also the explicit resolution of the gridscale transport of LEM quantities between neighboring grid boxes, which was absent in a previous approach that used the LEM as an LES SGS model in a cloud-physical application (Stechmann 2014). Ultimately, the supersaturation perturbation is considered in the diffusional growth of the superdroplets, enhancing or diminishing condensation and evaporation. Additionally, the new approach is shown to significantly mitigate the production of spurious supersaturations. The implementation of this new approach has been tested in two cases: an idealized two-dimensional bubble cloud and a well-known shallow cumulus intercomparison case. In all cases, an isotropic LES grid spacing of $20 \mathrm{~m}$ has been applied, which is usually considered to be too large to resolve all scales of inhomogeneous mixing.

In the initial stage of the bubble case, a relatively low intensity of turbulence resulted in strong SGS inhomogeneous mixing. As expected, this decreased the number of cloud droplets and increased the mean droplet radius. This resulted in an overall increase in the relaxation time scale and accordingly a slightly lower liquid water path compared to simulations without the new approach. Moreover, the new approach resulted in maximum radii of up to $26 \mu \mathrm{m}$ compared to $20 \mu \mathrm{m}$ using the standard approach, indicating that SGS inhomogeneous mixing would accelerate the production of precipitation embryos. Detailed analysis of superdroplet trajectories revealed that the largest droplets underwent significant SGS inhomogeneous condensational growth when the LEM was 

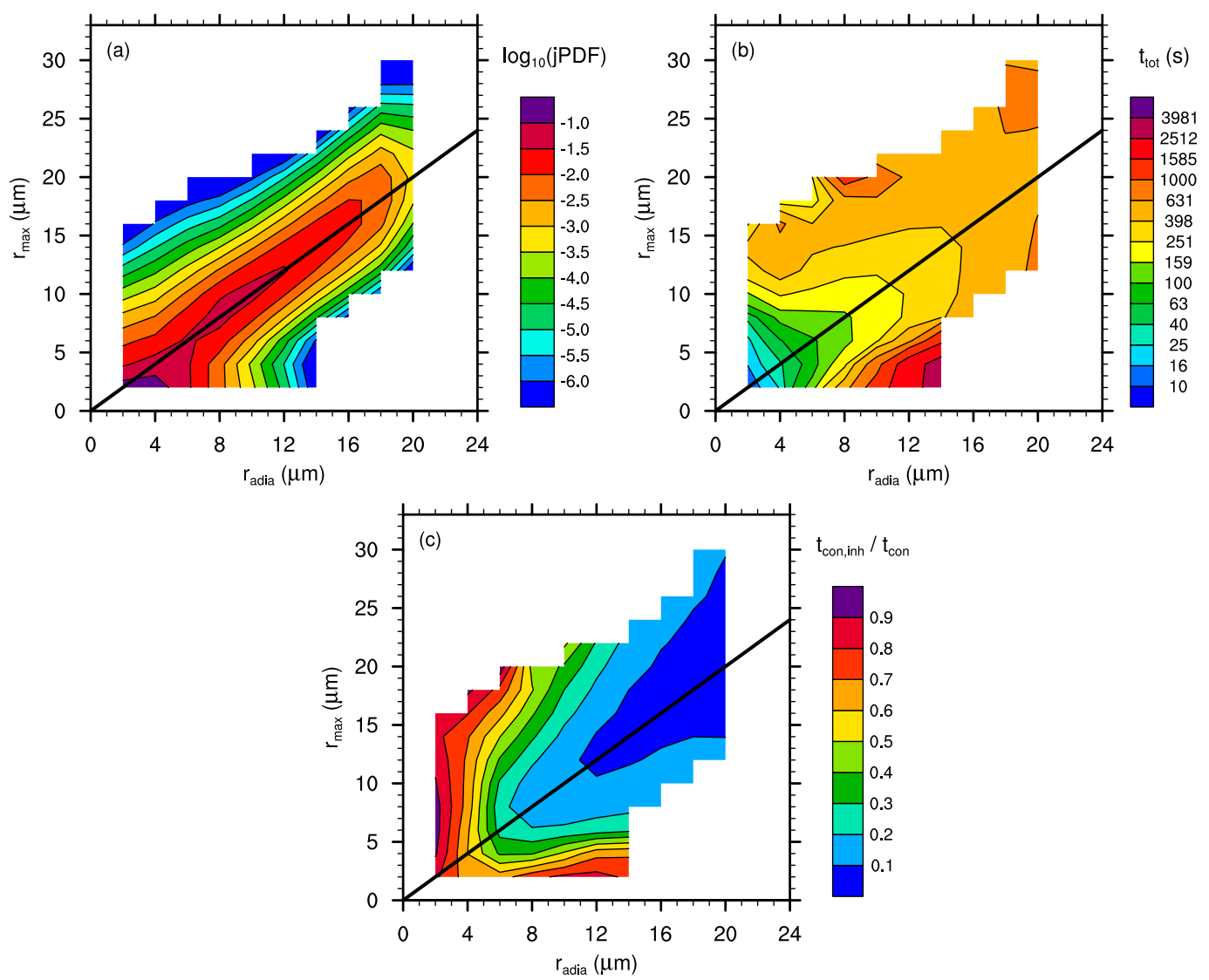

FIG. 11. (a) The joint PDF (jPDF) for the maximum radius $r_{\max }$ and (b) the corresponding adiabatic radius $r_{\text {adia }}$, as well as (c) the time spent between entrainment and reaching the maximum radius $t_{\text {tot }}$, and the time fraction of condensational growth in inhomogeneous conditions $\left(t_{\mathrm{con}, \text { inh }} / t_{\mathrm{con}}\right)$ as a function of $r_{\text {max }}$ and $r_{\text {adia }}$ for all superdroplets that have entered the cloud at its base $(<900 \mathrm{~m})$ within the LEM simulation. The thick black line marks adiabatic conditions $\left(r_{\max }=r_{\text {adia }}\right)$.

applied, while in the simulations without the LEM, the droplet in-cloud residence time was shown to be more important for the production of superadiabatic droplets.

The shallow cumulus case did not reveal significant differences in bulk properties derived from model runs with or without the new approach, which suggests that a grid spacing of $20 \mathrm{~m}$ might be sufficient to represent all relevant scales of inhomogeneous mixing for the analysis conducted here. Due to the highly turbulent nature of these clouds, the transition from inhomogeneous to homogeneous mixing takes place at relatively large spatial scales of about $1 \mathrm{~m}$, which is reported to favor homogeneous mixing (Lehmann et al. 2009) and corresponds to observations of turbulent cumuli by Jensen et al. (1985), Jensen and Baker (1989), and Gerber et al. (2008), which indicate homogeneous mixing. In fact, the largest impact of inhomogeneous mixing is only found at cloud base, where turbulence is weak, and not at higher levels of the cloud, which are usually targeted in observations. Nevertheless, inhomogeneous mixing is shown here to accelerate droplet growth at cloud base, whereas droplet in-cloud residence times are of primary importance for the production of the largest precipitation embryos at cloud top. Note, however, that the analysis presented here did not explicitly consider the cloud life cycle, in which mixing is reported to become more inhomogeneous toward the end (Schmeissner et al. 2015).

The results of this study suggest the following scenario for the initiation of rain. In highly turbulent cumulus clouds, the production of precipitation embryos might not benefit from inhomogeneous mixing since it is restricted to larger scales. Therefore, other processes need to be taken into account. Since droplet residence times are usually limited by the lifetime of the clouds, turbulenceenhanced collision rates, which tend to increase with turbulence, might be the primary path to raindrops for highly turbulent clouds (e.g., Devenish et al. 2012; Grabowski and Wang 2013). In less turbulent clouds (e.g., stratocumulus) inhomogeneous mixing might be of greater importance for the production of precipitation 

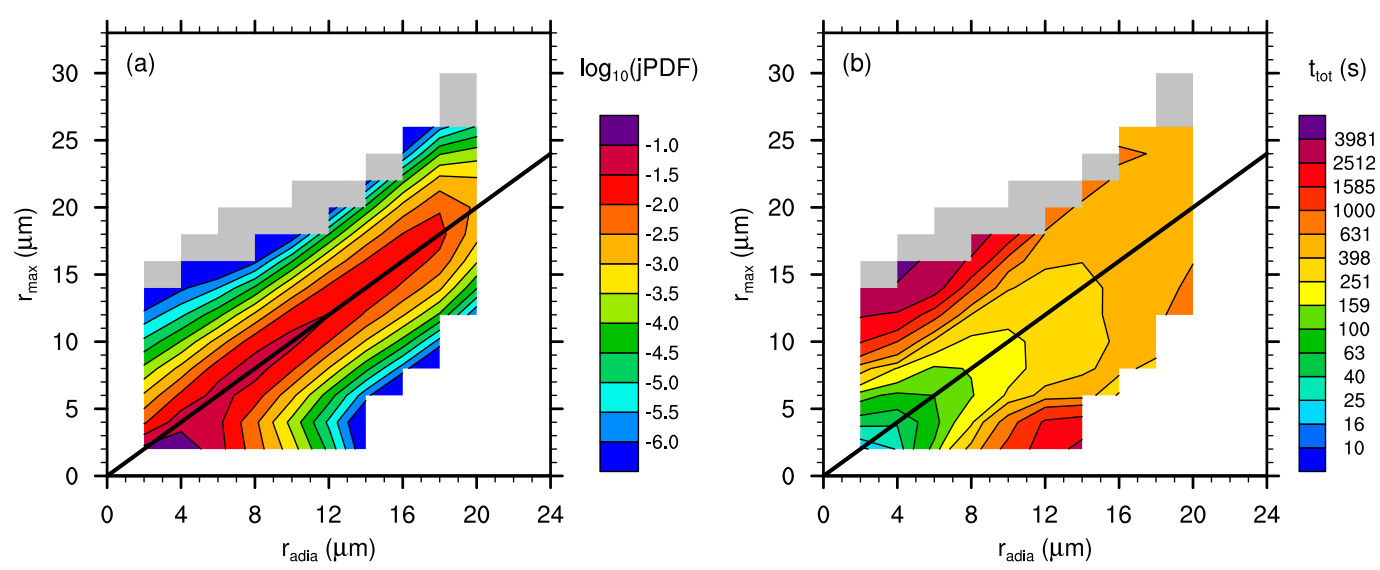

FIG. 12. (a) The joint PDF (jPDF) for the maximum radius $r_{\max }$ and (b) the corresponding adiabatic radius $r_{\text {adia }}$, as well as (b) the time spent between entrainment and reaching the maximum radius $t_{\text {tot }}$ as a function of $r_{\max }$ and $r_{\text {adia }}$ for all superdroplets that have entered the cloud at its base $(<900 \mathrm{~m})$ within the homogeneous simulation. The shaded area displays the range covered in the LEM simulation (see Fig. 11). The thick black line marks adiabatic conditions $\left(r_{\max }=r_{\text {adia }}\right)$.

embryos. However, these clouds also exhibit long droplet residence times (e.g., Feingold et al. 1996). Accordingly, these processes and their effects on the initiation of rain need to be evaluated in future studies.

Indeed, the simulation of stratocumulus is the next logical application for the presented approach since it not only allows for the investigation of inhomogeneous mixing in those clouds but it also mitigates the production of spurious supersaturations, which is a major numerical problem typically found just below the capping inversion, resulting in spurious activation of aerosols at cloud top (e.g., Stevens et al. 1996). Within this context, it will also be necessary to investigate aerosol effects on turbulence-microphysics interactions. In lowaerosol environments, turbulence-induced broadening of the droplet size distribution is enhanced due to the slower microphysical reaction time scale, which potentially accelerates rain formation (Chandrakar et al. 2016). In high-aerosol environments, turbulence is increased due to the so-called entrainment-evaporation feedback (Xue and Feingold 2006), which might result in a more homogeneous mixing scenario that decelerates rain formation (Feingold and Siebert 2009).

Moreover, the model itself needs to be extended for future applications since it a priori neglects sedimentation and collection. In fact, droplet sedimentation, a necessary prerequisite for collection, is prohibited because the current approach inherently demands that superdroplets move with their surrounding air. It is also able to enhance mixing, especially in low-turbulence environments (e.g., Su et al. 1998; Tölle and Krueger 2014). Therefore, it will be necessary to remove superdroplets from the LEM if they exhibit a significant sedimentation velocity, for example, based on the socalled velocity ratio between the sedimentation velocity and the (model's) Kolmogorov velocity scale (e.g., Vaillancourt and Yau 2000).

All in all, this study has shown that the use of the LEM as an SGS model is a useful addition to all warm-cloud LCMs, enabling these models to consider length scales that, to date, have been exclusive to direct numerical simulations.

Acknowledgments. This research was performed while FH held a visiting fellowship of the Cooperative Institute for Research in Environmental Sciences (CIRES) at the University of Colorado Boulder and the NOAA/Earth System Research Laboratory. Marat Khairoutdinov graciously provided the SAM model. We thank two anonymous reviewers and Steve Krueger for their comments.

\section{APPENDIX A}

\section{The Effect of LES Mixing on $\boldsymbol{\delta}_{n}^{\prime}$}

The Lagrangian tendency for the LES quantity of the absolute supersaturation $\bar{\delta}=\bar{q}_{v}-\bar{q}_{s}$, where $\bar{q}_{v}$ and $\bar{q}_{s}$ are the actual and saturation water vapor mixing ratios, respectively, is given by (e.g., Morrison and Grabowski 2008)

$$
\begin{aligned}
\frac{d \bar{\delta}}{d t}= & \left(\frac{\partial \bar{\delta}}{\partial t}\right)_{\text {lifting }}+\left(\frac{\partial \bar{\delta}}{\partial t}\right)_{\text {condensation }} \\
& +\left(\frac{\partial \bar{\delta}}{\partial t}\right)_{\text {mixing,LES }}+\left(\frac{\partial \bar{\delta}}{\partial t}\right)_{\text {other }} .
\end{aligned}
$$


Accordingly, $\bar{\delta}$ changes due to lifting (first term on the right-hand side), condensation and evaporation (second term), homogeneous mixing calculated in the LES's SGS model (third term), and possible other forcings (large-scale forcings and surface fluxes, as done in this study, but also radiation in future applications) (fourth term). For determining $\left(d \bar{\delta}_{n} / d t\right)_{\text {mixing,LES }}$ along the trajectory of individual superdroplets, Eq. (5) is rearranged and an index $n$ is added for each superdroplet:

$$
\begin{aligned}
\left(\frac{\partial \bar{\delta}_{n}}{\partial t}\right)_{\text {mixing,LES }}= & \frac{d \bar{\delta}_{n}}{d t}-\left(\frac{\partial \bar{\delta}_{n}}{\partial t}\right)_{\text {lifting }} \\
& -\left(\frac{\partial \bar{\delta}_{n}}{\partial t}\right)_{\text {condensation }}-\left(\frac{\partial \bar{\delta}_{n}}{\partial t}\right)_{\text {other }} .
\end{aligned}
$$

The terms on the right-hand side are then derived by tracking each superdroplet. The first term yields

$$
\frac{d \bar{\delta}_{n}}{d t}=\frac{\bar{\delta}\left[X_{n}(t)\right]-\bar{\delta}\left[X_{n}(t-\Delta t)\right]}{\Delta t}
$$

where $\bar{\delta}\left[X_{n}(t)\right]$ and $\bar{\delta}\left[X_{n}(t-\Delta t)\right]$ are the values of $\bar{\delta}$ at the position of the superdroplet at the end of the last time step and the current time step, respectively. The second term is determined by

$$
\left(\frac{\partial \bar{\delta}_{n}}{\partial t}\right)_{\text {lifting }}=\left(\frac{d q_{s}}{d T} \frac{g}{c_{p}}-\frac{\overline{q_{s}} \rho_{a} g}{p-e_{s}}\right) w_{n},
$$

where $d q_{s} / d T$ is the change in the saturation water vapor mixing ratio with temperature, which is given by the Clausius-Clapeyron relation; $g$ is the acceleration by gravity; $c_{p}$ is the specific heat capacity of air a constant pressure; $p$ is the hydrostatic pressure; and $e_{s}$ is the saturation water pressure. The vertical velocity of the superdroplet can be calculated from its vertical displacement during a time step:

$$
w_{n}=\frac{z_{n}(t)-z_{n}(t-\Delta t)}{\Delta t} .
$$

Since the two considered values of $z_{n}$ contain changes due to turbulent velocity fluctuations resulting from Eq. (1), the proposed model also includes supersaturation fluctuations caused by fluctuations in the vertical velocity similar to Grabowski and Abade (2017). However, their effects might be negligible here since a relatively small grid spacing is used for this study.

The last two terms of Eq. (5) are (usually) calculated for the entire grid box and need to be interpolated to the superdroplet. However, the determination of $\left(\partial \bar{\delta}_{n} / \partial t\right)_{\text {condensation }}$ by interpolation can be circumvented. This is advisable since condensation-evaporation might vary significantly across the cloud edge, potentially causing errors due to interpolation. To do so, $\bar{\delta}\left[X_{n}(t)\right]$ in Eq. (5) is evaluated before the calculation of condensation but after the calculation of dynamics (in the LES and the LCM). Since condensation has no direct effect on the dynamics during a time step (and hence SGS mixing), this procedure represents a consistent determination of $\left(\partial \bar{\delta}_{n} / \partial t\right)_{\text {mixing,LES }}$ without the need to consider $\left(\partial \bar{\delta}_{n} / \partial t\right)_{\text {condensation }}$ explicitly.

Note that the entire determination of $\left(\partial \bar{\delta}_{n} / \partial t\right)_{\text {mixing,LES }}$ as described above has the advantage of mitigating the production of spurious supersaturations, which is described in the following appendix B.

\section{APPENDIX B}

\section{Mitigation of Spurious Supersaturations}

Figure $3 \mathrm{c}$ indicates that spurious supersaturations are mitigated when the above-introduced approach for considering SGS inhomogeneous mixing is used. This can be understood by revisiting the idealized advection problem by Stevens et al. (1996), in which a cloud is advected from one grid box to the next (see their Fig. 2). In an LES, it is not possible to represent the location of the cloud edge on the SGS (i.e., in between grid boxes). Therefore, advection of the cloud into the initially cloud-free grid box changes the absolute supersaturation in the entire grid box according to

$$
\bar{\delta}(t)=\delta_{\text {free }}+\frac{\delta_{\text {cloud }}-\delta_{\text {free }}}{\tau_{\text {adv }}} t
$$

if there are no other effects on $\bar{\delta}$. Here, $\delta_{\text {free }}$ and $\delta_{\text {cloud }}$ are the initial values of the absolute supersaturation in the cloud-free and cloudy grid boxes, respectively, and $\tau_{\text {adv }}=\Delta x / u$ is the advection time scale determined by the length of the grid box $\Delta x$ and corresponding velocity $u$.

If a superdroplet moves from the cloudy to cloud-free grid box during one time step, it will experience the following change in $\bar{\delta}$ :

$$
\frac{d \bar{\delta}}{d t}=\frac{\bar{\delta}(\Delta t)-\delta_{\text {cloud }}}{\Delta t}
$$

In the new approach, this change is considered in the calculation of $(\partial \bar{\delta} / \partial t)_{\text {mixing,LES }}$ according to Eq. (5), in which $\delta_{n}^{\prime}$ is determined to counteract the nonadiabatic stimulus for evaporation and hence the mechanism for the subsequent production of spurious supersaturations.

As in Hoffmann (2016), the simplified advection problem by Stevens et al. (1996) is repeated using the 

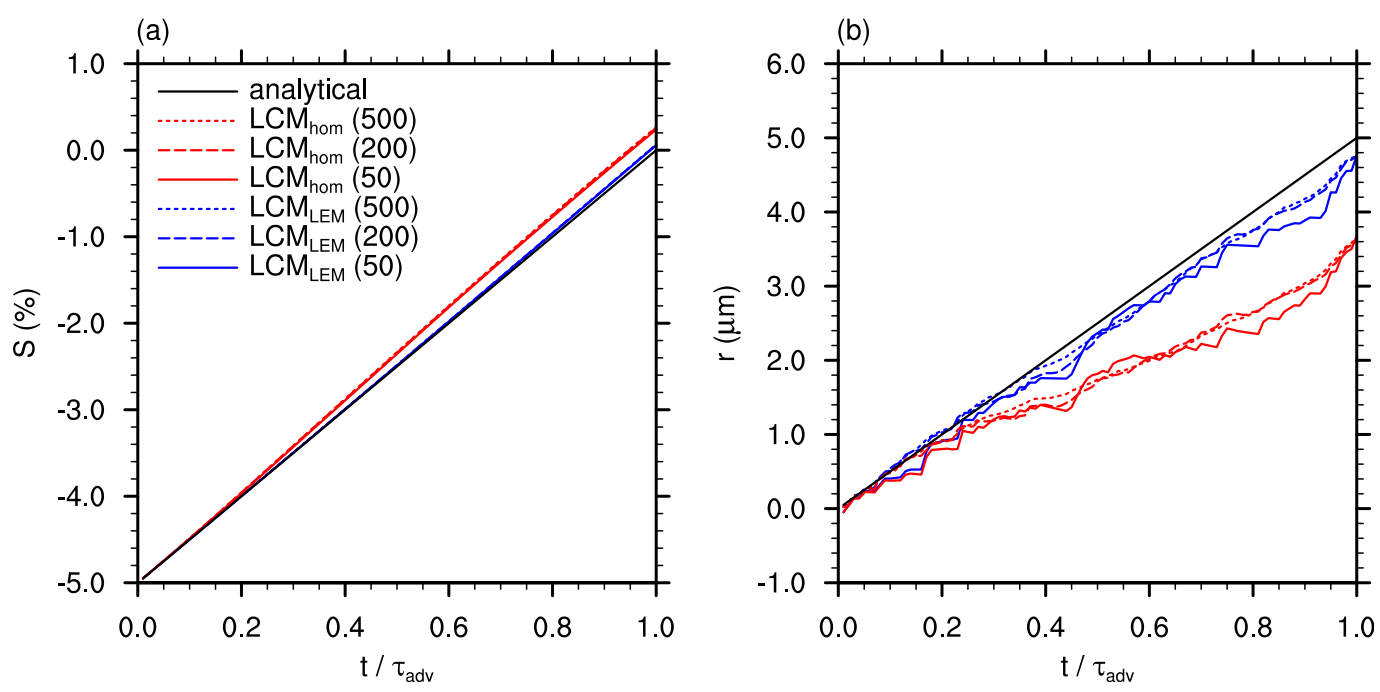

FIG. B1. Time series of (a) total relative supersaturation and (b) mean radius in the initially cloud-free grid box for simulations with ( $\mathrm{LCM}_{\mathrm{LEM}}$; blue lines) and without the new approach $\left(\mathrm{LCM}_{\mathrm{hom}}\right.$; red lines) for superdroplet concentrations of 50,200, and 500 superdroplets per grid box (line patterns).

LCM described above, neglecting vertical motion and mixing $\left[\left(\partial \delta_{n}^{\prime} / \partial t\right)_{\text {mixing,LEM }}=0\right]$. Note that $\left(\partial \bar{\delta}_{n} / \partial t\right)_{\text {mixing,LES }}$ will still be calculated from (5) using the first and third terms on the right-hand side of the equation. A unimodal distribution of cloud droplets with a radius of $5 \mu \mathrm{m}$ and a concentration of $30 \mathrm{~cm}^{-3}$ is initialized. The advection velocity is $u=0.1 \mathrm{~m} \mathrm{~s}^{-1}$ and $\Delta x=1 \mathrm{~m}$. Results from simulations with 50,200 , and 500 superdroplets per grid box with and without the new approach (blue and red lines, respectively) have been conducted. Figure B1 shows the total relative supersaturation and the mean radius in the initially cloud-free grid box. Simulations using the new approach exhibit a significantly reduced evaporation of droplets and hence an almost negligible production of spurious supersaturations compared to the simulations without the new approach. The reason for the slight deviation of the simulations with the new approach from the adiabatic solution is the third term in Eq. (4), which always depletes $\delta_{n}^{\prime}$ at a rate of $\delta_{n}^{\prime} / \tau_{n}$ irrespective of the true stimulus for evaporation and condensation expressed by $\bar{\delta}+\delta_{n}^{\prime}$ in Eq. (2). Accordingly, the ratio of the phase relaxation time scale $\tau_{n}$ to the advection time scale $\tau_{\text {adv }}$ steers the extent to which spurious evaporation and subsequently spurious supersaturations are mitigated by the new approach.

Note that a complete cancellation of spurious supersaturations would be possible within the current framework. For this, it is necessary to exclude the supersaturation perturbation $\delta_{n}^{\prime}$ from Eq. (3) as discussed above. This, however, distributes water and energy across LES and LCM and complicates their conservation, which is why this has been avoided here.

\section{APPENDIX C}

\section{Entraining Parcel Simulations}

Similar to the simulations presented in Su et al. (1998), the following analysis will present offline entraining parcel simulations (without a coupled LES), using the LEM implementation presented in section 2 (i.e., an LEM based on superdroplets and the perturbation supersaturation). Environmental conditions (e.g., background profiles of water vapor mixing ratio and potential temperature, as well as surface pressure) are all based on the shallow cumulus case by Siebesma et al. (2003) while Su et al. (1998) used a slightly different setup. All other parameters follow closely the latter study. The parcel is lifted with a vertical velocity of $2 \mathrm{~m} \mathrm{~s}^{-1}$ starting from the surface. The domain size and the model integral scale (i.e., the extent of the LEM) are set to $20 \mathrm{~m}$, and the kinetic energy dissipation rate is prescribed as $10 \mathrm{~cm}^{2} \mathrm{~s}^{-3}$. Entrainment is treated as a discrete process, in which a randomly selected, continuous region of the parcel is replaced by a blob of entrained environmental air. This region is randomly chosen, the size of the blob is specified to be $2 \mathrm{~m}$, and the timing of entrainment is randomized based on the fractional entrainment rate of $1.0 \mathrm{~km}^{-1}$. The model Kolmogorov length scale is determined by the number of simulated superdroplets [see Eq. (8)], with values set to 1.2, 0.6, 0.24, 0.24, 0.12, and $0.06 \mathrm{~m}$ when using $100,200,500,1000$, and 2000 superdroplets, respectively. Note that the entrained air contains as many superdroplets as the removed air, deprived of any liquid water, to maintain a constant particle concentration of $100 \mathrm{~cm}^{-3}$ throughout the simulation. 

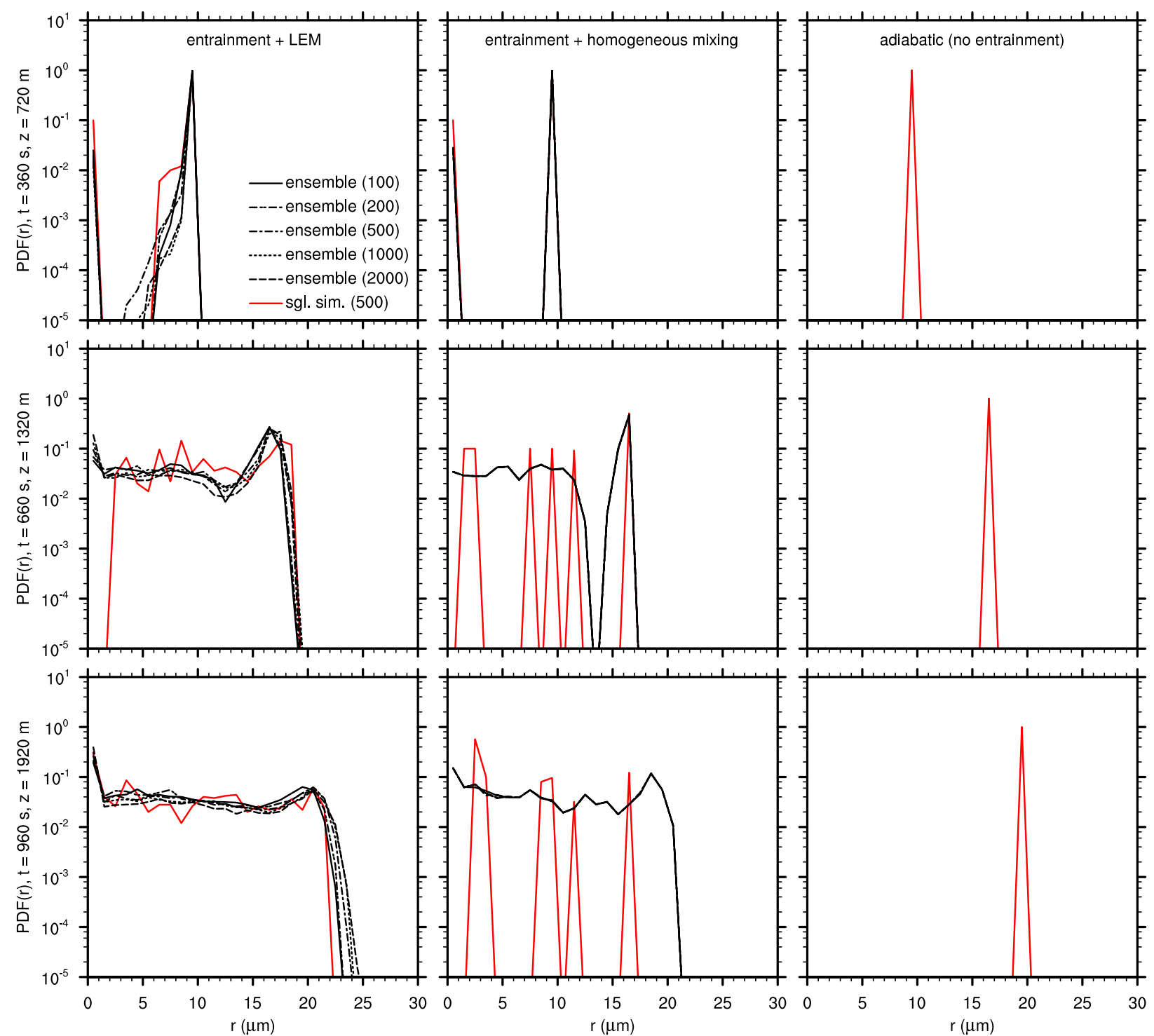

FIG. C1. PDFs of the droplet radius for entraining parcel simulations using the (left) LEM and (middle) instantaneous (homogeneous) mixing, as well as (right) a pure adiabatic parcel without entrainment at different times and heights during the parcel's ascent for ensembles simulations (black lines, with the dash pattern indicating the number of superdroplets) and individual simulations (red lines).

Figure $\mathrm{C} 1$ shows droplet size distributions from the entraining parcel using the LEM (left column) or instantaneous (homogeneous) mixing (center column), as well as a pure adiabatic parcel without entrainment (right column) similar to Fig. 3 in Su et al. (1998). Results from ensembles using 100 simulations (black lines) and individual simulations (red lines) are shown. Generally, the results agree well with Su et al. (1998). Especially in the individual simulations, the LEM is necessary to reproduce a realistic droplet spectrum. Moreover, the results show that entrainment generally results in larger maximum droplet sizes due to the reduction in the number of droplets in the parcel. This process is stronger when using the LEM and intensifies with the number of superdroplets and accordingly the model's ability to reproduce the physical Kolmogorov model results as closely as possible, as also described by Su et al. (1998). This has not been observed in the higher-dimensional simulations presented above, in which mixing can be represented on longer length scales due to the grid-scale transport between individual LEMs calculated for every grid box. 


\section{REFERENCES}

Abma, D., T. Heus, and J. P. Mellado, 2013: Direct numerical simulation of evaporative cooling at the lateral boundary of shallow cumulus clouds. J. Atmos. Sci., 70, 2088-2102, https:// doi.org/10.1175/JAS-D-12-0230.1.

Andrejczuk, M., J. M. Reisner, B. Henson, M. K. Dubey, and C. A. Jeffery, 2008: The potential impacts of pollution on a nondrizzling stratus deck: Does aerosol number matter more than type? J. Geophys. Res., 113, D19204, https://doi.org/10.1029/ 2007JD009445.

Baker, M., and J. Latham, 1979: The evolution of droplet spectra and the rate of production of embryonic raindrops in small cumulus clouds. J. Atmos. Sci., 36, 1612-1615, https://doi.org/ 10.1175/1520-0469(1979)036<1612:TEODSA > 2.0.CO;2.

- R. Corbin, and J. Latham, 1980: The influence of entrainment on the evolution of cloud droplet spectra: I. A model of inhomogeneous mixing. Quart. J. Roy. Meteor. Soc., 106, 581598, https://doi.org/10.1002/qj.49710644914.

Beals, M. J., J. P. Fugal, R. A. Shaw, J. Lu, S. M. Spuler, and J. L. Stith, 2015: Holographic measurements of inhomogeneous cloud mixing at the centimeter scale. Science, 350, 87-90, https://doi.org/10.1126/science.aab0751.

Bodenschatz, E., S. P. Malinowski, R. A. Shaw, and F. Stratmann, 2010: Can we understand clouds without turbulence? Science, 327, 970-971, https://doi.org/10.1126/science.1185138.

Boffetta, G., and R. E. Ecke, 2012: Two-dimensional turbulence. Annu. Rev. Fluid Mech., 44, 427-451, https://doi.org/10.1146/ annurev-fluid-120710-101240.

Chandrakar, K. K., W. Cantrell, K. Chang, D. Ciochetto, D. Niedermeier, M. Ovchinnikov, R. A. Shaw, and F. Yang, 2016: Aerosol indirect effect from turbulence-induced broadening of cloud-droplet size distributions. Proc. Natl. Acad. Sci. USA, 113, 14243-14248, https://doi.org/10.1073/ pnas.1612686113.

Cooper, W. A., S. G. Lasher-Trapp, and A. M. Blyth, 2013: The influence of entrainment and mixing on the initial formation of rain in a warm cumulus cloud. J. Atmos. Sci., 70, 1727-1743, https://doi.org/10.1175/JAS-D-12-0128.1.

Cuijpers, J., and P. Duynkerke, 1993: Large eddy simulation of trade wind cumulus clouds. J. Atmos. Sci., 50, 3894-3908, https://doi.org/ 10.1175/1520-0469(1993)050<3894:LESOTW>2.0.CO;2.

Deardorff, J. W., 1980: Stratocumulus-capped mixed layers derived from a three-dimensional model. Bound.-Layer Meteor., 18, 495-527, https://doi.org/10.1007/BF00119502.

Devenish, B., and Coauthors, 2012: Droplet growth in warm turbulent clouds. Quart. J. Roy. Meteor. Soc., 138, 1401-1429, https://doi.org/10.1002/qj.1897.

Feingold, G., and H. Siebert, 2009: Cloud-aerosol interactions from the micro to the cloud scale. Clouds in the Perturbed Climate System: Their Relationship to Energy Balance, Atmospheric Dynamics, and Precipitation, J. Heintzenberg and R. Charlson, Eds., The MIT Press, 319-338, https://doi.org/ 10.7551/mitpress/9780262012874.003.0014.

_ - W. Cotton, B. Stevens, and A. Frisch, 1996: The relationship between drop in-cloud residence time and drizzle production in numerically simulated stratocumulus clouds. J. Atmos. Sci., 53, 1108-1122, https://doi.org/10.1175/1520-0469(1996)053<1108: TRBDIC $>2.0 . \mathrm{CO} ; 2$.

Gerber, H. E., G. M. Frick, J. B. Jensen, and J. G. Hudson, 2008: Entrainment, mixing, and microphysics in trade-wind cumulus. J. Meteor. Soc. Japan, 86A, 87-106, https://doi.org/10.2151/ jmsj.86A.87.
Götzfried, P., B. Kumar, R. A. Shaw, and J. Schumacher, 2017: Droplet dynamics and fine-scale structure in a shearless turbulent mixing layer with phase changes. J. Fluid Mech., 814, 452-483, https://doi.org/10.1017/jfm.2017.23.

Grabowski, W. W., and T. L. Clark, 1991: Cloud-environment interface instability: Rising thermal calculations in two spatial dimensions. J. Atmos. Sci., 48, 527-546, https://doi.org/ 10.1175/1520-0469(1991)048<0527:CIIRTC > 2.0.CO;2.

_ environment. Annu. Rev. Fluid Mech., 45, 293-324, https://doi.org/ 10.1146/annurev-fluid-011212-140750.

— through eddy hopping: Turbulent adiabatic parcel simulations. J. Atmos. Sci., 74, 1485-1493, https://doi.org/10.1175/ JAS-D-17-0043.1.

_, P. Dziekan, and H. Pawlowska, 2018: Lagrangian condensation microphysics with Twomey CCN activation. Geosci. Model Dev., 11, 103-120, https://doi.org/10.5194/gmd-11-103-2018.

Hill, A. A., G. Feingold, and H. Jiang, 2009: The influence of entrainment and mixing assumption on aerosol-cloud interactions in marine stratocumulus. J. Atmos. Sci., 66, 1450-1464, https:// doi.org/10.1175/2008JAS2909.1.

Hoffmann, F., 2016: The effect of spurious cloud edge supersaturations in Lagrangian cloud models: An analytical and numerical study. Mon. Wea. Rev., 144, 107-118, https://doi.org/ 10.1175/MWR-D-15-0234.1.

— 2017: On the limits of Köhler activation theory: How do collision and coalescence affect the activation of aerosols? Atmos. Chem. Phys., 17, 8343-8356, https://doi.org/10.5194/ acp-17-8343-2017.

- - H. Siebert, J. Schumacher, T. Riechelmann, J. Katzwinkel, B. Kumar, P. Götzfried, and S. Raasch, 2014: Entrainment and mixing at the interface of shallow cumulus clouds: Results from a combination of observations and simulations. Meteor. Z., 23, 349-368, https://doi.org/10.1127/0941-2948/2014/0597.

- S. Raasch, and Y. Noh, 2015: Entrainment of aerosols and their activation in a shallow cumulus cloud studied with a coupled LCM-LES approach. Atmos. Res., 156, 43-57, https:// doi.org/10.1016/j.atmosres.2014.12.008.

— Y. Yoh, and S. Raasch, 2017: The route to raindrop formation in a shallow cumulus cloud simulated by a Lagrangian cloud model. J. Atmos. Sci., 74, 2125-2142, https://doi.org/10.1175/ JAS-D-16-0220.1.

Holland, J. Z., and E. M. Rasmusson, 1973: Measurement of atmospheric mass, energy, and momentum budgets over a 500-kilometer square of tropical ocean. Mon. Wea. Rev., 101, 44-55, https://doi.org/10.1175/1520-0493(1973)101<0044: MOTAME $>2.3 . \mathrm{CO} ; 2$.

Jarecka, D., W. W. Grabowski, and H. Pawlowska, 2009: Modeling of subgrid-scale mixing in large-eddy simulation of shallow convection. J. Atmos. Sci., 66, 2125-2133, https://doi.org/ 10.1175/2009JAS2929.1.

Jensen, J. B., and M. B. Baker, 1989: A simple model of droplet spectral evolution during turbulent mixing. J. Atmos. Sci., 46, 2812-2829, https://doi.org/10.1175/1520-0469(1989)046<2812: ASMODS $>2.0$.CO;2.

— P. H. Austin, M. B. Baker, and A. M. Blyth, 1985: Turbulent mixing, spectral evolution and dynamics in a warm cumulus cloud. J. Atmos. Sci., 42, 173-192, https://doi.org/10.1175/15200469(1985)042<0173:TMSEAD>2.0.CO;2.

Jiang, H., H. Xue, A. Teller, G. Feingold, and Z. Levin, 2006: Aerosol effects on the lifetime of shallow cumulus. Geophys. Res. Lett., 33, L14806, https://doi.org/10.1029/2006GL026024. 
Kerstein, A. R., 1988: A linear-eddy model of turbulent scalar transport and mixing. Combust. Sci. Technol., 60, 391-421, https://doi.org/10.1080/00102208808923995.

Khairoutdinov, M. F., and D. A. Randall, 2003: Cloud resolving modeling of the ARM summer 1997 IOP: Model formulation, results, uncertainties, and sensitivities. J. Atmos. Sci., 60, 607-625, https:// doi.org/10.1175/1520-0469(2003)060<0607:CRMOTA > 2.0.CO;2.

Kogan, Y. L., 1991: The simulation of a convective cloud in a 3-D model with explicit microphysics. Part I: Model description and sensitivity experiments. J. Atmos. Sci., 48 , 1160-1189, https://doi.org/10.1175/1520-0469(1991)048<1160: TSOACC $>2.0 . \mathrm{CO} ; 2$.

Krueger, S. K., 1993: Linear eddy modeling of entrainment and mixing in stratus clouds. J. Atmos. Sci., 50, 3078-3090, https://doi.org/ 10.1175/1520-0469(1993)050<3078:LEMOEA > 2.0.CO;2.

- 2016: Representing the effects of entrainment and mixing on warm-cloud microphysics in large-eddy simulations. 2016 Fall Meeting, San Francisco, CA, Amer. Geophys. Union, Abstract A33I-0374.

— C.-W. Su, and P. A. McMurtry, 1997: Modeling entrainment and finescale mixing in cumulus clouds. J. Atmos. Sci., 54, 2697-2712, https://doi.org/10.1175/1520-0469(1997)054<2697: MEAFMI $>2.0 . \mathrm{CO} ; 2$.

Kumar, B., J. Schumacher, and R. A. Shaw, 2013: Cloud microphysical effects of turbulent mixing and entrainment. Theor. Comput. Fluid Dyn., 27, 361-376, https://doi.org/10.1007/ s00162-012-0272-z.

,-- , and — 2014: Lagrangian mixing dynamics at the cloudy-clear air interface. J. Atmos. Sci., 71, 2564-2580, https://doi.org/10.1175/JAS-D-13-0294.1.

Lasher-Trapp, S. G., W. A. Cooper, and A. M. Blyth, 2005: Broadening of droplet size distributions from entrainment and mixing in a cumulus cloud. Quart. J. Roy. Meteor. Soc., 131, 195-220, https://doi.org/10.1256/qj.03.199.

Lehmann, K., H. Siebert, and R. A. Shaw, 2009: Homogeneous and inhomogeneous mixing in cumulus clouds: Dependence on local turbulence structure. J. Atmos. Sci., 66, 3641-3659, https://doi.org/10.1175/2009JAS3012.1.

Menon, S., and A. R. Kerstein, 2011: The linear-eddy model. Turbulent Combustion Modeling. Fluid Mechanics and Its Applications, T. Echekki and E. Mastorakos, Eds., Springer Series on Fluid Mechanics and Its Applications, Vol. 95, Springer, 221-247.

Mordy, W., 1959: Computations of the growth by condensation of a population of cloud droplets. Tellus, 11, 16-44, https://doi.org/ 10.3402/tellusa.v11i1.9283.

Morrison, H., and W. W. Grabowski, 2008: Modeling supersaturation and subgrid-scale mixing with two-moment bulk warm microphysics. J. Atmos. Sci., 65, 792-812, https://doi.org/ 10.1175/2007JAS2374.1.

Riechelmann, T., Y. Noh, and S. Raasch, 2012: A new method for large-eddy simulations of clouds with Lagrangian droplets including the effects of turbulent collision. New J. Phys., 14, 065008, https://doi.org/10.1088/1367-2630/14/6/065008.

Rogers, R. R., and M. K. Yau, 1989: A Short Course in Cloud Physics. Pergamon Press, 293 pp.

Schmeissner, T., R. Shaw, J. Ditas, F. Stratmann, M. Wendisch, and H. Siebert, 2015: Turbulent mixing in shallow trade wind cumuli: Dependence on cloud life cycle. J. Atmos. Sci., 72,14471465, https://doi.org/10.1175/JAS-D-14-0230.1.

Shaw, R. A., 2003: Particle-turbulence interactions in atmospheric clouds. Annu. Rev. Fluid Mech., 35, 183-227, https://doi.org/ 10.1146/annurev.fluid.35.101101.161125.

Shima, S.-I., K. Kusano, A. Kawano, T. Sugiyama, and S. Kawahara, 2009: The super-droplet method for the numerical simulation of clouds and precipitation: A particle-based and probabilistic microphysics model coupled with a non-hydrostatic model. Quart. J. Roy. Meteor. Soc., 135, 1307-1320, https://doi.org/ 10.1002/qj.441.

Siebesma, A. P., and Coauthors, 2003: A large eddy simulation intercomparison study of shallow cumulus convection. J. Atmos. Sci., 60, 1201-1219, https://doi.org/10.1175/15200469(2003)60<1201:ALESIS > 2.0.CO;2.

Sölch, I., and B. Kärcher, 2010: A large-eddy model for cirrus clouds with explicit aerosol and ice microphysics and Lagrangian ice particle tracking. Quart. J. Roy. Meteor. Soc., 136, 2074-2093, https://doi.org/10.1002/qj.689.

Stechmann, S. N., 2014: Multiscale eddy simulation for moist atmospheric convection: Preliminary investigation. J. Comput. Phys., 271, 99-117, https://doi.org/10.1016/j.jcp.2014.02.009.

Stevens, B., R. L. Walko, W. R. Cotton, and G. Feingold, 1996: The spurious production of cloud-edge supersaturations by Eulerian models. Mon. Wea. Rev., 124, 1034-1041, https://doi. org/10.1175/1520-0493(1996)124<1034:TSPOCE > 2.0.CO;2.

Su, C.-W., S. K. Krueger, P. A. McMurtry, and P. H. Austin, 1998: Linear eddy modeling of droplet spectral evolution during entrainment and mixing in cumulus clouds. Atmos. Res., 4748, 41-58, https://doi.org/10.1016/S0169-8095(98)00039-8.

Tölle, M. H., and S. K. Krueger, 2014: Effects of entrainment and mixing on droplet size distributions in warm cumulus clouds. J. Adv. Model. Earth Syst., 6, 281-299, https://doi.org/10.1002/ 2012MS000209.

Vaillancourt, P. A., and M. Yau, 2000: Review of particleturbulence interactions and consequences for cloud physics. Bull. Amer. Meteor. Soc., 81, 285-298, https://doi.org/10.1175/ 1520-0477(2000)081<0285:ROPIAC $>2.3 . C O ; 2$.

Weil, J. C., P. P. Sullivan, and C.-H. Moeng, 2004: The use of largeeddy simulations in Lagrangian particle dispersion models. J. Atmos. Sci., 61, 2877-2887, https://doi.org/10.1175/JAS3302.1 .

Xue, H., and G. Feingold, 2006: Large-eddy simulations of trade wind cumuli: Investigation of aerosol indirect effects. J. Atmos. Sci., 63, 1605-1622, https://doi.org/10.1175/ JAS3706.1. 